\title{
SCHWARZ REFLECTION PRINCIPLE IN COMPLEX SPACES OF DIMENSION TWO
}

\author{
Xiaojun Huang ${ }^{1}$ \\ Department of Mathematics \\ The University of Chicago \\ Chicago, IL 60637
}

Contents

$\S 0$. Introduction

$\S 1$. Preliminaries, notation, and definitions

$\S 2$. Basic approaches and preliminary facts

$\S 3$. Extension across $M_{1}^{s}$

$\S 4$. Extension across $C_{t}^{+}$

$\S 5$. Completion of the proof of the Main TheoremAssuming Theorem C

$\S 6$. Statement of Theorem D and related notation

$\S 7$. Extension of $G(f, \lambda)$-Proof of Theorem D(a)

$\S 8$. Branches of $\mathcal{F}$ and Segre varieties

$\S 9$. Branch locus of $\mathcal{F}$ and Segre varieties

$\S 10$. A preservation principle for $\mathcal{F}$-Proof of Theorem D (b)

$\S 11$. Completion of the proof of Theorem C

\footnotetext{
${ }^{1}$ Supported in part by NSF DMS-9500881 and research at MSRI is supported in part by NSF DMS-9022140.
}

Typeset by $\mathcal{A} \mathcal{M}-\mathrm{TEX}_{\mathrm{E}}$ 


\section{XIAOJUN HUANG}

\section{$\S 0$. Introduction}

Let $M_{1}$ and $M_{2}$ be two real analytic hypersurfaces in $\mathbf{C}^{n}$, which do not contain any non-trivial complex analytic varieties. Let $f$ be a continuous CR-mapping from $M_{1}$ into $M_{2}$ (i.e, each component of $f$ is annihilated, in the sense of distributions, by the induced CR vector fields of $M_{1}$ ). It has been an open question of long standing whether $f$ must be real analytic over all of $M_{1}$. In the present paper, we will give a solution to this question in the case of complex dimension 2. More precisely, we will prove the following

Main Theorem. Let $M_{1}$ and $M_{2}$ be two real analytic hypersurfaces in $\mathbf{C}^{2}$, which do not contain any non-trivial complex analytic variety. Let $f$ be a continuous $C R$ mapping from $M_{1}$ into $M_{2}$. Then $f$ is real analytic at each point of $M_{1}$.

This result seems new even under the additional assumption that $f$ is smooth over $M_{1}$, or the hypersurfaces are pseudoconvex (see questions asked in [7], [8], [13]). (However, see [35] in case the hypersurfaces are strongly pseudoconvex).

Our approach is heavily based on some recently developed tools for performing reflections for mappings between real analytic hypersurfaces ([38], [39], [24], [1], [14], [6], [19], [22]). The question of holomorphic extensions for CR mappings between real analytic hypersurfaces has attracted considerable attention since the work of Fefferman [25]. For more recent work, see [31], [34], [10], [3], [1], [6], [19], [14], [20], [35], [22], [4], [30], [23], and the references therein, as well as the survey paper by Forstneric [26]. We note that, in the papers cited above, there is always a certain kind of assumption requiring the "biholomorphic" or "proper holomorphic" extension of the maps to one side, or requiring some sort of unique continuation properties (or the Hopf lemma property) for the maps under study. In the 2-dimensional case, studied in this paper, we will deal with the general CR mappings. Recent work on the existence of inner functions reveals that there are many $L^{\infty}$ - CR mappings between spheres in $\mathbf{C}^{2}$ which are not real analytic at any point. Also, it is easy to construct a smooth but not real analytic CR mapping between real 


\section{SCHWARZ REFLECTION PRINCIPLE}

analytic hypersurfaces of infinite type. Hence, our result is optimal and can be viewed as a natural extension of the classical Schwarz reflection principle to the complex space of dimension 2 .

We recall that a real analytic hypersurface, which does not contain any non trivial complex analytic variety, is said to be of finite type in the sense of D'Angelo [18], (or simply finite type in the sense of Kohn, in the $\mathbf{C}^{2}$ case). We also recall a result proved in [9] and [36] which states that when the real analytic hypersurface $M$ is of D'Angelo finite type, a function over $M$ is CR if and only if it can be extended holomorphically to a certain side of $M$. Hence our main theorem can be stated as follows. (In fact, it is in this form that we will prove our Main Theorem.)

Theorem A. Let $M_{1}$ and $M_{2}$ be two real analytic hypersurfaces of finite type in $\mathbf{C}^{2}$. Let $D$ be a bounded domain in $\mathbf{C}^{2}$ such that $D \cup M_{1}$ forms a manifold with smooth boundary $M_{1}$. Assume that $f$ is a holomorphic mapping from $D$ into $\mathbf{C}^{2}$, that is continuous up to $D \cup M_{1}$ and maps $M_{1}$ into $M_{2}$. Then $f$ admits a holomorphic extension across $M_{1}$. Moreover, when $f$ is not constant, then the extension of $f$ is locally proper and preserves the sides of the hypersurfaces (near $M_{1}$ ).

We mention that one of the main features in Theorem $\mathrm{A}$ is that $f$ is not assumed apriori to be proper from $D$. As is known, it is always a difficult problem to understand when a CR mapping can be realized as the boundary value of a certain proper holomorphic map.

As an immediate application of Theorem A and some known results of [20], we have the following

Corollary B. Let $D_{1}$ and $D_{2}$ be two bounded real analytic domains in $\mathbf{C}^{2}$. Let $f$ be a proper holomorphic mapping from $D_{1}$ to $D_{2}$, that is continuous up to $\overline{D_{1}}$. Then $f$ admits a holomorphic extension across $\overline{D_{1}}$. In addition, when the boundaries of $D_{1}$ and $D_{2}$ are algebraic, the assumption of continuity of $f$ up to the boundary can be dropped.

The above corollary was obtained in the author's previously circulated preprint [29]. The proof of Corollary B presented in [29] is based on a reg- 


\section{XIAOJUN HUANG}

ularity result of holomorphic correspondences first proved in [29] (which is our present Theorem D to be stated in $\S 6$ ), and is based on the construction of holomorphic correspondences for proper holomorphic maps using some ideas on the application of Bishop's extension lemma as in [23]. When the map $f$ is biholomorphic, then Corollary B is studied in the work of [22] and [23]. In [23], Diederich-Pinchuk showed that any biholomorphic mapping between bounded real analytic domains in $\mathbf{C}^{2}$ can be extended holomorphically across the boundary. Their proof uses a regularity theorem of holomorphic correspondences for biholomorphic maps proved in [20] and uses the Bishop extension lemma to construct the desired correspondences. We also mention that, when $D_{1}$ and $D_{2}$ are pseudoconvex, Corollary B was settled by Baouendi-Bell-Rothschild in [1].

The organization of the paper is as follows: The paper is divided into two major parts. Part I consists of $\S 2-\S 5$. We will prove, in this part, the main theorem assuming a regularity result of holomorphic correspondences for general CR-mappings (i.e. Theorem $\mathrm{C}$ to be stated in $\S 2$ ). In $\S 1$, we introduce some basic tools and notation which will be used throughout the paper. In $\S 2$, we discuss our basic approaches and some known results which will be used from $\S 2$ to $\S 5$. In $\S 3$, we prove holomorphic extension across strongly pseudoconvex points. $\S 4$ is devoted to the extension across a large piece of weakly pseudoconvex points. In $\S 5$, we extend the mapping along a thin set and therefore complete the argument for Part I.

Part II is devoted to the proof of the above mentioned regularity result for holomorphic correspondences (i.e, the proof of Theorem C). In fact, we will prove Theorem $\mathrm{D}$, to be stated in $\S 6$, which is slightly more general than Theorem C. (A related version of this theorem was obtained in [20] in case the map under study is a CR homeomorphism, by using a completely different method). We first prove, in $\S 7$, the extension theorem for a reflection function (see the related notion in [3] and [1]), by using the weak version of the edge of the wedge theorem. In $\S 8$ and $\S 9$, we connect the branching locus of the holomorphic correspondences, constructed from the assumption of Theorem $\mathrm{D}$, with the invariant varieties. In $\S 10$, we prove the Baouendi-Rothschild 


\section{SCHWARZ REFLECTION PRINCIPLE}

Hopf lemma in our setting, using ideas appearing in [7]. In $§ 11$, we complete the proof of Theorem D by using the lemmas established in the preceding sections and by applying an argument of Hartogs extension type.

Acknowledgment: The author is grateful to S. Baouendi, L. Rothschild, and S. Webster for their constant help and encouragement during the preparation of this work. He thanks S. Li, S. Krantz, R. Narasimhan, Y. Pan, and S. Pinchuk, for helpful discussions. Also, he is indebted to P. Ebenfelt for his very careful reading and many comments, which greatly improved both the exposition and the mathematics in the paper.

Note added February, 1996: This paper is the revised version of the author's previously circulated preprint [29], whose main results included our present Corollary B, the algebraic version of Theorem A, and, in particular, Theorem $D$ to be stated in $\S 6$ here. We would like to mention that this paper owes certain ideas to the above cited work of Webster [39], BaouendiBell-Rothschild [1], Baouendi-Rothschild [7], and Diederich-Fornaess-Ye [22]. The proper mapping version of the regularity result of holomorphic correspondences (closely related to Theorem 1.1 in [29], or Theorem D in the present version ) was also announced by Diederich-Pinchuk at MSRI in November, 1995. See also [23], where a different construction of holomorphic correspondences for biholomorphisms was presented by using the known regularity theorem of holomorphic correspondences for biholomorphisms proved in [20]. (However, we mention that the work in [23] does not seem to be relevant and useful to the study of regularity problems of holomorphic correspondences itself.)

\section{$\S 1$. Preliminaries, Notation, and Definitions}

Let $M$ be a real analytic hypersurface of finite D'Angelo type in $\mathbf{C}^{n+1}$. For each point $p \in M$, there exist a small open neighborhood $U^{(p)}$ of $p$, a real analytic function $\rho(z, \bar{w})$ over $U^{(p)} \times U^{(p)}$ such that $M=\left\{z \in U^{(p)}\right.$ : $\rho(z, \bar{z})=0\}$ and $\left.\mathrm{d} \rho\right|_{M} \neq 0$. For each $z \in U^{(p)}$, as in [38], we call the analytic 


\section{XIAOJUN HUANG}

variety $Q_{z}=\left\{w \in U^{(p)}: \rho(w, \bar{z})=0\right\}$ the Segre variety of $M$, associated to $z$. By shrinking $U^{(p)}$, we can choose two neighborhoods $\widetilde{\mathcal{P}}$ and $\mathcal{P}$ of $p$ such that the following holds (see [19] or [22]): (i) $\mathcal{P} \subset \subset \widetilde{\mathcal{P}} \subset \subset U^{(p)}$; (ii) for each

$z \in \overline{\mathcal{P}}, Q_{z} \cap \overline{\widetilde{\mathcal{P}}}$ is a simply connected submanifold with smooth boundary, and $Q_{z}$ is transversal to $\partial \widetilde{\mathcal{P}}$; (iii) $\mathcal{P} \backslash M$ has two connected components, homeomorphic to the ball. Here, for a subset $A \subset \mathbf{C}^{n}$ we write $\bar{A}$ for its closure in $\mathbf{C}^{n}$.

For each $z \in \overline{\mathcal{P}}$, write $A_{z}=\left\{w \in \overline{\mathcal{P}}: Q_{w}=Q_{z}\right\}$. By the finite D'Angelo type assumption of $M$ and by shrinking $U^{(p)}$ if necessary, we can always assume that $\# A_{z}<\infty$ for each $z \in \overline{\mathcal{P}}$ and $A_{p}=\{p\}$ (see [3] and [24]).

Still letting $p \in M$, we can always find a biholomorphic change of coordinates [3] such that $p=0$ and $M$ in $U^{(p)}$ is defined by an equation of the following form :

$$
\rho(z, \bar{z})=z_{n+1}+\overline{z_{n+1}}+\sum_{j} \phi_{j}\left(z^{\prime}, \overline{z^{\prime}}\right)\left(\operatorname{Im} z_{n+1}\right)^{j}
$$

or

$$
\widetilde{\rho}(z, \bar{z})=z_{n+1}+\overline{z_{n+1}}+\sum_{j} \widetilde{\phi_{j}}\left(z^{\prime}, \overline{z^{\prime}}\right)\left(z_{n+1}\right)^{j},
$$

where $z^{\prime}=\left(z_{1}, \cdots, z_{n}\right), \phi_{j}$ 's and $\widetilde{\phi}_{j}$ 's do not contain any harmonic terms, and $\rho$ is real-valued. In what follows, we will call such a coordinate system normal for $M$ near $p=0$, and call $\rho$ or $\widetilde{\rho}$ the normalized defining equation of $M$. Notice that in the normal coordinate system, $\left(0^{\prime},-t\right) \in Q_{\left(0^{\prime}, t\right)}$ for each $t \in \mathbf{R}$ near the origin. More generally, by varying the base point $p$, and by noticing the smooth dependence of the normal coordinates with respect to $p([3])$, we can find a smooth conjugating operator $\mathcal{R}: \widetilde{\mathcal{P}} \rightarrow U^{(0)}$ such that $\mathcal{R}(z) \in Q_{z}, \mathcal{R}$ reverses the sides of $M$ and $\left.\mathcal{R}\right|_{M}=$ id. (See ([22], pp 545) for a similar notion). For more properties concerning Segre varieties, we refer the reader to [19], [22].

Now, we let $M$ be in $\mathbf{C}^{2}$ and let $\Omega$ be a bounded domain such that $\Omega \cup M$ forms a manifold with smooth boundary $M$. From $\S 2$ to $\S 5$, we need the following semi-analytic stratification for $M$ as introduced in [22]:

$$
M=M^{s} \cup M^{a} \cup C_{t}^{+} \cup C_{t}^{-} \cup C_{t}^{ \pm} \cup C_{o} .
$$




\section{SCHWARZ REFLECTION PRINCIPLE}

Here

(a) $M^{s}\left(M^{a}\right)$ is the set of strongly pseudoconvex (strongly pseudoconcave, respectively) boundary points of $\Omega \cup M$;

(b) $C_{t}^{+}\left(C_{t}^{-}\right)$is a locally finite union of 2-dimensional totally real analytic submanifolds, where the vanishing order of the Levi form of $M$, as the boundary of $\Omega \cup M$, is even, locally constant, and positive (negative, respectively). Therefore, $\Omega$ is pseudoconvex near $C_{t}^{+}$and pseudoconcave near $C_{t}^{-}$, and any holomorphic function defined over $\Omega$ can be extended holomorphically across each point of $C_{t}^{-}$;

(c) $C_{t}^{ \pm}$is a locally finite union of 2-dimensional totally real submanifolds, where the vanishing order of the Levi form is odd and locally constant. It is known (see [9], or [21], [2]) that any CR function defined near $C_{t}^{ \pm}$can be holomorphically extended to both sides of $M$ near $C_{t}^{ \pm}$;

(d) $C_{o}$ is a locally finite union of one dimensional real analytic curves and isolated points.

In what follows, we write $W L(M)=M \backslash\left(M^{s} \cup M^{a}\right)$.

\section{$\S 2$. Basic Approaches and Some Preliminary Facts}

From now on until $\S 5$, we let $M_{1}$ and $M_{2}$ be two connected real analytic hypersurfaces of finite type in $\mathbf{C}^{2}$. Let $f$ be a non-constant continuous $\mathrm{CR}$ mapping from $M_{1}$ into $M_{2}$. For a given point $p \in M_{1}$, write $D$ for the side of $M_{1}$ near $p$, into which any CR function near $p$ (in particular, the components of $f$ ) extends holomorphically. We notice that $D$ (near $p$ ) can be filled in by 'small' analytic disks attached to $M_{1}([9][36])$. Let $q=f(p)$. As introduced in $\S 1$, we have the following stratification for $M_{1}$ with respect to $D: M_{1}=M_{1}^{s} \cup M_{1}^{a} \cup C_{t}^{+} \cup C_{t}^{-} \cup C_{t}^{ \pm} \cup C_{o}$.

In all that follows, for convenience, we use the notation in $\S 1$ for $M_{1}$ and add 'prime' for those corresponding to $M_{2}$.

For a point $p \in M_{1}$ and a totally real analytic submanifold $S \subset M_{1}$ of real dimension 2 with $p \in S$, after a holomorphic change of coordinates, we 


\section{XIAOJUN HUANG}

can always assume that $p=0, S$ is a small open neighborhood of $0 \in \mathbf{R}^{2}$, and $y_{2}=\operatorname{Im} z_{2}$ is the outer normal direction of $M_{1}$ at $p=0$. Write $D^{c}=$ $U^{(0)} \backslash\left(D \cup M_{1}\right)$, and $\mathcal{W}^{ \pm}=\left\{\left(z_{1}, z_{2}\right): \pm \operatorname{Im} z_{2}>\left|\operatorname{Im} z_{1}\right|\right\}$ for the standard wedges with edge $S$. Then near $p=0, \mathcal{W}^{+} \cap \mathcal{P} \subset D^{c}, \mathcal{W}^{-} \cap \mathcal{P} \subset D$. Moreover, we can assume that $\mathcal{W}^{+} \cap \mathcal{P}$ is connected.

A closed subset $E \subset \mathcal{W}^{+} \cap \mathcal{P}$ is said to be negligible if $\mathcal{W}^{+} \cap \mathcal{P} \backslash E$ is a connected open dense subset of $\mathcal{W}^{+} \cap \mathcal{P}$ and $S \backslash \bar{E}$ is dense in $S$.

In the following, we always assume that $\mathcal{P}$ and $\mathcal{P}^{\prime}$ are sufficiently small neighborhoods of $p=0 q=0$, respectively.

We now introduce the following:

Definition 2.1. $f$ is said to extend as a holomorphic correspondence to $\left(\mathcal{W}^{+} \cap \mathcal{P} ; \mathcal{P}^{\prime}\right)$ if for some negligible set $E$ of $\mathcal{P} \cap \mathcal{W}^{+}$, there is a complex analytic variety $\mathcal{V} \subset\left(\mathcal{P} \cap \mathcal{W}^{+} \backslash E\right) \times \mathcal{P}^{\prime}$ such that (i) the natural projection $\pi$ from $\mathcal{V}$ to $\mathcal{P} \cap \mathcal{W}^{+} \backslash E$ is finite to one; (ii) the restriction of $\pi$ to each irreducible component of $\mathcal{V}$ with complex dimension 2 is surjective; and (iii) there exists a sequence $\left\{p_{j}\right\} \subset S$, converging to $p=0$, so that $f$ extends biholomorphically across each $p_{j}$ and $\mathcal{V}$ contains the graph of $f$ over $O\left(p_{j}\right) \cap \mathcal{W}^{+} \cap \mathcal{P} \backslash E$.

The starting point for the proof of our theorem is the following regularity result of holomorphic correspondences, whose proof will be the main content of Part II of this paper:

Theorem C. Let $M_{1}, M_{2}, f, p=q=f(p)=0, S, \mathcal{W}^{+}$be as above. Suppose that for some sufficiently small $\mathcal{P}$ and $\mathcal{P}^{\prime}, f$ extends as a holomorphic correspondence to $\left(\mathcal{W}^{+} \cap \mathcal{P} ; \mathcal{P}^{\prime}\right)$. Also suppose that $f$ extends almost everywhere across $M_{1}$. Then $f$ admits a holomorphic extension across $p$.

By the above Theorem $\mathrm{C}$ and a Hopf type lemma obtained in [7] (see the following Lemma $2.2(\mathrm{~h})$ ), to prove the Main Theorem, it suffices for us to show the existence of the analytic variety $\mathcal{V}$ as in Definition 2.1. Indeed, this will be our approach (see already related approaches in [39], [19], [22], [23]).

In the rest of this section, we describe briefly how $\mathcal{V}$ can be constructed. Then we list some known facts to be used later. 


\section{SCHWARZ REFLECTION PRINCIPLE}

First, for each $z \in \mathcal{P} \cap \mathcal{W}^{+}$, we let $Q_{z}^{c}$ be the connected component of $Q_{z} \cap D \cap \widetilde{\mathcal{P}}$ which contains $\bar{z}$ (see already [22] for some related notion). (In the special coordinates where $S=\mathbf{R}^{2} \cap O(0)$, it is easy to see that $\bar{z}$ is indeed in $\left.Q_{z} \cap D\right)$. Here and in what follows, we use the notation $O(a)$ to denote a small open neighborhood of $a$, whose size might be different in different contexts. Sometimes, we write $O_{z}(a)$ for a small neighborhood of $a$ in the space where the $z$-coordinates are used.

For each point $a \in Q_{z} \cap U^{(0)}$, we will write ${ }_{a} Q_{z}$ for the germ of $Q_{z}$ at $a$. As usual, we denote by $J_{f}$ the Jacobian of $f$. Write $E_{0}=\left\{z \in \mathcal{P} \cap \mathcal{W}^{+}\right.$: $\left.J_{f}(\bar{z})=0\right\}$. Assume that $E$ is a negligible set of $\mathcal{P} \cap \mathcal{W}^{+}$. Motivated by the invariant property of Segre varieties (see also related work in [39], and, in particular, the work in [22]), we define

$$
\mathcal{V}(E)=\left\{(z, w) \in\left(\mathcal{P} \cap \mathcal{W}^{+} \backslash E\right) \times \mathcal{P}^{\prime}: f\left(Q_{z}^{c}\right) \subset Q_{w}^{\prime}\right\}
$$

Define $\pi\left(\pi^{\prime}\right.$, respectively) to be the natural projection from $\mathcal{V}(E)$ into $\left(\mathcal{W}^{+} \cap\right.$ $\mathcal{P} \backslash E)\left(\mathcal{P}^{\prime}\right.$, respectively). Write $\operatorname{Bih}_{M_{1}}(f)$ for the collection of points in $M_{1}$ where $f$ extends biholomorphically.

In the following lemma, we collect some facts, most of which are, more or less, implied in the existing work or can be proved easily. Also, in its statement, we keep the previously established notation.

Lemma 2.2. Assume that $\operatorname{Bih}_{M_{1}}(f)$ is dense in $M_{1}$ and $\operatorname{Bih}_{M_{1}}(f) \cap S$ is dense in $S$. Also assume that $E$ contains $E_{0}$ as defined above. Then the following holds:

(a) $\pi$ is finite to one.

(b) $\mathcal{V}(E)$ is a closed analytic variety of dimension at most 2.

(c) Let $\left(z_{0}, w_{0}\right) \in \mathcal{V}(E)$ and let $\left(z_{j}, w_{j}\right) \subset \mathcal{V}(E)$ be a sequence such that $\lim \left(z_{j}, w_{j}\right)=\left(z_{0}, w_{0}\right)$. If $\mathcal{V}(E)$ has dimension 2 at each $\left(z_{j}, w_{j}\right)$, then $\left(z_{0}, w_{0}\right)$ is also a point of dimension 2 of $\mathcal{V}(E)$.

(d) Suppose that $p_{0} \in(S \backslash \bar{E}) \cap \operatorname{Bih}_{M_{1}}(f)$. Then $\mathcal{V}(E)$ contains:

$$
\left.\Gamma_{f}\right|_{O\left(p_{0}\right) \cap \mathcal{W}^{+} \cap \mathcal{P} \backslash E}=\left\{(z, w) \in\left(O\left(p_{0}\right) \cap \mathcal{W}^{+} \cap \mathcal{P} \backslash E\right) \times \mathcal{P}^{\prime}: w=f(z)\right\} .
$$




\section{XIAOJUN HUANG}

(Hence, $\mathcal{V}(E)$ is a complex analytic variety of dimension 2, by the hypothesis and the statement in (b)).

(e) [22] Let $E^{*}=\left\{z \in \mathcal{P} \cap \mathcal{W}^{+} \backslash E_{0}: f\left(Q_{z}^{c}\right) \subset Q_{w}^{\prime}, w \in W L\left(M_{2}\right)\right\}$. Then $E^{*}$ is a locally real subanalytic set of real dimension at most 2 in $\mathcal{W}^{+} \cap \mathcal{P} \backslash E_{0}$. Here, as before, $W L\left(M_{2}\right)$ is the set of points where the Levi form of $M_{2}$ vanishes.

(f) Let $p \in M_{1}^{s}$. After shrinking $\mathcal{P}$ and $\widetilde{\mathcal{P}}$, if necessary, then for each $z \in$ $\mathcal{P} \cap D^{c}, Q_{z} \cap \widetilde{\mathcal{P}} \cap D=Q_{z}^{c}$. Moreover, $Q_{z}^{c}$ is simply connected and the diameter of $Q_{z}^{c}$ goes to 0 when $z \rightarrow p$.

(g) Let $q=f(p) \in M_{2}^{s} \cup M_{2}^{a}$. Then $f$ maps $D$ to the pseudoconvex side of $M_{2}$ near $q$. Also, $Q_{q}^{\prime} \cap O(q)$ stays in the pseudoconcave side of $M_{2}$.

$(h)([7])$ Assume that $f$ extends holomorphically across $p$. Then $f$ is locally finite to one and proper near $p$. Moreover, the normal component of $f$ has non-vanishing derivative along the normal direction of $M_{1}$ at $p$.

(i) Assume that for each small $\mathcal{P}^{\prime}$, when $\widetilde{\mathcal{P}}$ is sufficiently small, $\pi$ becomes surjective. Then $f$ extends as a holomorphic correspondence $\mathcal{V}(E)$ to $\left(\mathcal{W}^{+} \cap\right.$ $\mathcal{P} ; \mathcal{P}^{\prime}$ ) for some small $\mathcal{P}$ and $\mathcal{P}^{\prime}$.

Proof of Lemma 2.2: First of all, since $E \supset E_{0}$, we know that $f$ is not constant when restricted to $Q_{z}^{c}$ for each $z \in \mathcal{P} \cap \mathcal{W}^{+} \backslash E_{0}$. In particular, by noting the finiteness of the set $A_{w}^{\prime}$, this implies that for each point $z \in$ $\mathcal{P} \cap \mathcal{W}^{+} \backslash E_{0}$, there are only finitely many $w^{\prime}$ s so that $f\left(Q_{z}^{c}\right) \subset Q_{w}^{\prime}$. (In fact, all these points have the same associated Segre varieties; for they all have a piece in common.) This proves that $\pi$ is finite to one. Similarly, one can show that $\pi^{\prime}$ is also locally finite to one. (2.b) follows from a straightforward verification (see [22]). (2.c) follows easily from (2.a), (2.b), and some basic properties of analytic varieties. (2.d) is an easy corollary of the invariant property of Segre varieties. (2.e) follows from the facts that $E^{*}=\pi\left(\pi^{\prime}-1\left(W L\left(M_{2}\right)\right)\right.$, $\pi, \pi^{\prime}$ are locally finite to one, and $W L\left(M_{2}\right)$ is a real analytic subset of real dimension at most 2 (see the related explanation in [22] and also [17]). (2.f) was essentially proved in [39]. The first part of (2.g) is an easy application of the maximum principle and the disk filling-in property of $D$. The last 


\section{SCHWARZ REFLECTION PRINCIPLE}

part of (2.g) also follows from the work of [39]. (2.h) is the content of the Baouendi-Rothschild Hopf lemma [7]. (2.i) follows from the definition of holomorphic correspondences and the above mentioned fact that all points in $\pi^{-1}(z)$ correspond to the same Segre variety.

We next present an easy fact, whose statement, in particular, implies that $f$ extends holomorphically across an open dense subset of $M_{1}$.

Lemma 2.3. Let $p \in M_{1}^{s}$. For any totally real analytic submanifold $S \subset M_{1}$ of dimension 2 through $p$, $f$ admits a holomorphic extension across a dense open subset of $S$ near $p$.

Proof of Lemma 2.3: First, by applying a result of Pinchuk-Tsyganov [35] and by applying the reflection principle, we know that $f$ extends across each open subset of $S$, whose image is contained in either $M_{2}^{s} \cup M_{2}^{a}$ or some totally real analytic piece of $W L\left(M_{2}\right)$ of dimension 2. Also notice that any open subset of $S$ cannot be mapped into any one dimensional arc in $C_{o}^{\prime}$, by using the reflection principle and by noting the fact that the Jacobian of $f$ is not zero on a dense open subset of $S$ (see [8]). Meanwhile, it is easy to see that any open piece of $S$ cannot be mapped into isolated points in $C_{o}^{\prime}$; for $f$ is not constant. Hence, the proof is complete.

Before leaving this section, we mention that the construction of holomorphic correspondences by using Segre varieties was implied in the work of Webster [39], where a simple proof of Fefferman's extension theorem was presented in the real analytic category and a continuity method was employed to obtain the required correspondence. Related approaches were further explored in the work [19], [20], [22], [23] (see, in particular, the work in [22]). Our construction of $\mathcal{V}(E)$, though largely motivated by the above mentioned work of [39] and [22], is different in many aspects from the existing constructions and seems, in particular, suitable for the study of general CR mappings. 


\section{XIAOJUN HUANG}

\section{Part I. Proof of the Main Theorem Assuming Theorem C}

In this part, we assume Theorem $\mathrm{C}$ and give a complete proof of our Main Theorem (in the form of Theorem A). Our approach is, as described above, to construct the variety $\mathcal{V}$ as in Definition (2.1). We first observe that $f$ already admits holomorphic extension across the pseudoconcave part $C_{t}^{-} \cup C_{t}^{ \pm} \cup M_{1}^{a}$ of $M_{1}$, by the discussions in $\S 1$.

\section{$\S 3$. Extension across $M_{1}^{s}$}

We keep the notation which we have set up so far. We will prove the following result in this section:

Lemma 3.1. $f$ extends holomorphically across $M_{1}^{s}$.

Proof of Lemma 3.1: Let $p=0 \in M_{1}^{s}$. Choose $S$ and its wedges $\mathcal{W}^{ \pm}$as before. Also, we let $E=E_{0} \cup E^{*}$, where $E^{*}$ is the same as defined in Lemma 2.2 (e). $E \backslash E_{0}$ is locally a real subanalytic subset of real dimension at most 2. Moreover, by using Lemma 2.3 and the work in [8], one can easily show that $S \backslash \bar{E}$ is dense in $S$. Hence, one can easily verify that $E$ is a negligible set of $\mathcal{W}^{+} \cap \mathcal{P}$.

Define $\mathcal{V}(E)$ as in (2.1). Then, by Lemma 2.3, Theorem C, and Lemma 2.2 (i), we need only to show that for a small neighborhood $\mathcal{P}^{\prime}$ of $q=0$, after making $\widetilde{\mathcal{P}}$ sufficiently small, then $\pi$ is surjective.

Seeking a contradiction, suppose not. Let $p_{0} \in(S \backslash \bar{E}) \cap \operatorname{Bih}_{M_{1}}(f)$ be sufficiently close to 0 .

Write $\mathcal{V}^{p_{0}}(E)$ for the 2-dimensional component of $\mathcal{V}(E)$, which contains the graph of $f$ over $O\left(p_{0}\right) \cap \mathcal{W}^{+} \cap \mathcal{P} \backslash E$. By our assumption, $\pi: \mathcal{V}^{p_{0}}(E) \rightarrow$ $\mathcal{W}^{+} \cap \mathcal{P} \backslash E$ is not surjective either. On the other hand, we notice that $\left.\pi\right|_{\mathcal{V}^{p_{0}}(E)}$ is an open mapping, for it is a local analytic covering map. Hence, we can find a curve $\gamma:(0,1] \rightarrow \mathcal{W}^{+} \cap \mathcal{P} \backslash E$ starting from $p_{0}$ (i.e, $p_{0}=\lim _{t \rightarrow 0^{+}} \gamma(t)$ ) such that $\gamma(1) \notin \pi\left(\mathcal{V}^{p_{0}}\right)$, and $\gamma((0,1)) \subset \pi\left(\mathcal{V}^{p_{0}}\right)$. Moreover, after perturbing slightly $\gamma(t)$ to avoid the branch locus of $\mathcal{V}^{p_{0}}(E)$, we can assume that there is 


\section{SCHWARZ REFLECTION PRINCIPLE}

a unique lift of $\gamma(t)$ to $\widetilde{\gamma}(t) \subset \mathcal{V}^{p_{0}}(t \in(0,1))$ such that $\widetilde{\gamma}(0)=\left(p_{0}, f\left(p_{0}\right)\right)$ and $\widetilde{\gamma}(t)=(\gamma(t), w(t))$ for $t \in(0,1)$. Therefore, $f\left(Q_{\gamma(t)}^{c}\right) \subset Q_{w(t)}^{\prime}$ for $t \in(0,1)$. Meanwhile, by Lemma $2.2(\mathrm{~d})$, we have for each small $t, \mathcal{R}^{\prime}(w(t)) \in f\left(Q_{\gamma(t)}^{c}\right)$. For simplicity, we write $w^{*}(t)=\mathcal{R}^{\prime}(w(t))$ in what follows:

Claim 3.2. $w^{*}(t) \in f\left(Q_{\gamma(t)}^{c}\right)$ for $t \in(0,1)$.

Assume the claim for the moment. Then, for each $t$, we can find some $\xi(t) \in Q_{\gamma(t)}^{c}$ such that $f(\xi(t))=w^{*}(t)\left(=\mathcal{R}^{\prime}(w(t))\right.$. Notice that $|f(\xi(t))| \leq$ $\max _{w \in f\left(\partial Q_{\gamma(t)}^{c}\right)}|w|$, by the maximum principle. After shrinking $\mathcal{P}$, we can assume that $w^{*}(t) \in \mathcal{P}^{\prime *}$, where $\mathcal{P}^{\prime *} \subset \subset \mathcal{P}^{\prime}$ and $\mathcal{R}^{\prime}\left(\mathcal{P}^{\prime *}\right) \subset \subset \mathcal{P}^{\prime}$. By passing to the limit, we see that all limit points of $w(t)$ as $t \rightarrow 1^{-}$stay in $\mathcal{P}^{\prime *}$.

On the other hand, for each limit point, say $w(1)$, of $w(t)$ as $t \rightarrow 1^{-}$, one sees that $f\left(Q_{\gamma(1)}^{c}\right) \subset Q_{w(1)}^{\prime}$. Hence $(z(1), w(1)) \in \mathcal{V}(E)$. By Lemma 2.2 (c), it follows that $(z(1), w(1)) \in \mathcal{V}^{p_{0}}$. This is a contradiction.

Therefore, to complete the proof of Lemma 3.1, we need only prove Claim 3.2:

Let $\delta=\max \left\{\tau \in(0,1]:\right.$ for $\left.t \in(0, \tau), w^{*}(t) \in f\left(Q_{\gamma(t)}^{c}\right)\right\}$. Then $\delta>0$ by the above observation. Suppose that $\delta<1$. Then there exists a point $\xi(t) \in$ $Q_{\gamma(t)}^{c}$ for each $t \in(0, \tau)$, such that $f(\xi(t))=w^{*}(t)$. Now, we have two cases to study: (i) $\lim _{t_{j} \rightarrow \delta^{-}} \xi\left(t_{j}\right)=\xi_{0} \in M_{1}$; and (ii) $\lim _{t_{j} \rightarrow \delta^{-}} \xi\left(t_{j}\right)=\xi_{0} \notin M_{1}$ for some subsequence $\left\{t_{j}\right\}$ converging to $\delta$.

In Case (i), we have $w(\delta)=w^{*}(\delta)=f\left(\xi_{0}\right) \in M_{2}$. Hence, $f\left(Q_{\gamma(\delta)}^{c} \cap\right.$ $\left.O\left(\xi_{0}\right)\right) \subset Q_{w(\delta)}^{\prime} \cap O(w(\delta))$. Since, by our choice of $E, f$ is not constant on $Q_{\gamma(\delta)}^{c}$ and $w(\delta) \notin W L\left(M_{2}\right)$, we can easily reach a contradiction by applying the maximum principle and Lemma $2.2(\mathrm{~g})$.

Therefore, Case (ii) is the only possibility. After taking a limit, it then holds that $w^{*}(\delta) \in f\left(Q_{\gamma(\delta)}^{c}\right)$.

Next, since $\delta<1$, there exists a sequence $t_{j} \rightarrow \delta^{+}$such that $w^{*}\left(t_{j}\right) \notin$ $f\left(Q_{\gamma\left(t_{j}\right)}^{c}\right)$. Now, for $t$ sufficiently close to $\delta$ and some small $\epsilon\left(\epsilon^{\prime}\right.$, respectively), we can easily find a conformal mapping $\phi_{t}\left(\psi_{t}\right.$, respectively), depending continuously on $t$, from the unit disk $\Delta$ to $Q_{\gamma(t)}^{c} \cap B_{\epsilon}\left(\xi_{0}\right)$ (from $Q_{w(t)}^{\prime} \cap$ $B_{\epsilon^{\prime}}\left(w^{*}(\delta)\right)$ to $\Delta$, respectively). Here, $\epsilon$ is suitably smaller than $\epsilon^{\prime}$ and $B_{a}(b)$ 


\section{XIAOJUN HUANG}

denotes the ball centered at $b$ with radius $a$. Since $\psi_{\delta} \circ f \circ \phi_{\delta}$ is not constant, by the Hurwitz theorem and by noticing that $\psi_{t_{j}} \circ f \circ \phi_{t_{j}}-\psi_{t_{j}}\left(w^{*}\left(t_{j}\right)\right) \neq 0$, it follows that $w^{*}(\delta) \notin f\left(Q_{\gamma(\delta)}^{c} \cap B_{\epsilon}\left(\xi_{0}\right)\right)$. This is a contradiction. The proof of Claim 3.2 and, thus the proof of Lemma 3.1, is complete.

\section{$\S 4$. Extension across $C_{t}^{+}$}

We now use Lemma 3.1 to construct the holomorphic extension across $C_{t}^{+}$. Our proof depends strongly on the fact that $\mathcal{V}(E)$ is spread over the wedge $\mathcal{W}^{+}$, which avoids the difficulty arising from the points close to strongly pseudoconvex pieces of $M_{1}$.

Lemma 4.1. Let $p \in M_{1}$. Assume that the following holds: (i) $f$ admits an extension across $Q_{p} \cap M_{1} \cap \overline{\widetilde{\mathcal{P}}} \backslash\{p\}$ and (ii) $f(p) \in M_{2}^{s} \cup M_{2}^{a}$. Then $f$ admits a holomorphic extension across $p$.

We mention here that the importance of having a nice property similar to $f \in C^{\omega}\left(Q_{p} \cap M_{1} \cap \overline{\widetilde{\mathcal{P}}} \backslash\{p\}\right)$ to start with was first showed up in the work of $[22]$.

Proof of Lemma 4.1: In the proof of this lemma, we let $E=E_{0}=\{z \in$ $\left.\mathcal{W}^{+} \cap \mathcal{P}: J_{f}(\bar{z})=0\right\}$ and let $S$ be an edge with $S \cap M_{1}^{s}$ open dense in $S$. After shrinking $\widetilde{\mathcal{P}}$, we can assume, by the hypothesis, the existence of a small $\epsilon$, such that $f$ extends holomorphically to the union of the balls $B_{\epsilon}\left(p_{j}\right)$ of radius $\epsilon$ with center at $p_{j} \in Q_{p} \cap \partial \widetilde{\mathcal{P}} \cap \bar{D}$, where $\left\{p_{j}\right\}$ is a finite set obtained from the open covering lemma (or, an empty set in case $Q_{p} \cap \bar{D} \cap \partial \widetilde{\mathcal{P}}=\emptyset$ ).

By the continuity principle, we can assume that $Q_{p} \backslash\{p\} \not \subset D$ near $p$; for otherwise, $f$ extends automatically across $p$. By Lemma $2.2(\mathrm{~h})$ and the choice of the balls $B_{\epsilon}\left(p_{j}\right)$, it thus follows easily that $f$ is not constant when restricted to each $B_{\epsilon}\left(p_{j}\right) \cap Q_{p}$ (provided it is not an empty set).

As before, we still denote by $\pi$ the natural projection from $\mathcal{V}(E)$ to $\mathcal{W}^{+} \cap \mathcal{P} \backslash E$. Suppose that $\pi$ is not surjective, no matter how we shrink 


\section{SCHWARZ REFLECTION PRINCIPLE}

the size of $\mathcal{P}$. Then for each small $\delta$, it is clear that there exists a sequence $\left\{\left(z_{j}(\delta), w_{j}(\delta)\right)\right\} \in \mathcal{V}(E)$ such that $z_{j}(\delta) \rightarrow p,\left|w_{j}(\delta)\right|=\delta$, and $\lim w_{j}(\delta)=w(\delta)$ for some point $w(\delta)$ with $|w(\delta)|=\delta$.

By making $\widetilde{\mathcal{P}}$ sufficiently small, we have $f(\widetilde{\mathcal{P}} \cap D) \subset \subset \mathcal{P}^{*} * \subset B_{\delta}(0) \subset \subset$ $\mathcal{P}^{\prime}$, where $B_{\delta}(0)$ is the ball with center 0 and radius $\delta$. Then we claim that $\overline{Q_{z_{j}}^{c}} \cap \partial \widetilde{\mathcal{P}} \neq \emptyset$ for $j>>1$. Suppose not. Notice that $\left.f\right|_{Q_{z_{j}}^{c}}$ is a locally proper map from $Q_{z_{j}}^{c}$ into $Q_{w_{j}(\delta)}^{\prime}$. We can then conclude that $f\left(Q_{z_{j}}^{c}\right)$ will contain the connected piece of $Q_{w_{j}(\delta)}^{\prime}$ which stays in the pseudoconvex side of $M_{2}$, by Lemma $2.2(\mathrm{~g})$. Therefore, by Lemma $2.2(\mathrm{f}), w_{j}^{*}(\delta) \in f\left(Q_{z_{j}}^{c}\right)$ and thus $w_{j}^{*}(\delta) \in \mathcal{P}^{\prime *}$. This gives a contradiction, once we make $\mathcal{P}^{\prime *}$ sufficiently small so that $\mathcal{R}^{\prime}\left(\mathcal{P}^{\prime *}\right) \subset \subset B_{\delta}(0)$.

Hence, we can find $\xi_{j} \in \overline{Q_{z_{j}}^{c}} \cap \partial \widetilde{\mathcal{P}}$ with $\xi_{j} \rightarrow \xi_{0} \in \partial \widetilde{\mathcal{P}} \cap Q_{p} \cap \bar{D}$ as $j \rightarrow \infty$. Thus we have $f\left(O\left(\xi_{0}\right) \cap Q_{p}\right) \subset Q_{w(\delta)}^{\prime}$. Notice that $f$ is not constant when restricted to each of $Q_{p} \cap B_{\epsilon}\left(p_{j}\right)^{\prime}$ s, by the above observation. It thus follows that $Q_{w(\delta)}^{\prime}$ is uniquely determined by the ball $B_{\epsilon}\left(p_{j}\right)$ which contains $\xi_{0}$. Hence, there are only finitely many choices of such $Q_{w(\delta)}^{\prime}$ 's. This, together with the finiteness of $A_{w}^{\prime}$ for $w \in \mathcal{P}^{\prime}$, contradicts the infinitely many choices of such $w(\delta)$ 's.

Lemma 4.2. Assume that $f$ admits a holomorphic extension across an open dense subset of $C_{t}^{+}$. Then $f$ extends holomorphically across each point in $C_{t}^{+}$.

Proof of Lemma 4.2: Let $p \in C_{t}^{+}$and let $S=C_{t}^{+} \cap O(p)$. As before, after a holomorphic change of coordinates, we assume that $p=0, S=\mathbf{R}^{2} \cap O(0)$. Let $E=E_{0}$. Then the assumption in the lemma indicates that $E$ is a negligible set of $\mathcal{W}^{+} \cap \mathcal{P}$ and $\mathcal{V}(E)$ is an analytic variety of dimension 2 . Hence, as before, to finish the proof of Lemma 4.2, it suffices for us to show that $\pi$ is surjective after making $\mathcal{P}$ sufficiently small.

If this is not the case, as in Lemma 4.1, for each small $\delta>0$, there is a sequence $z_{j}(\delta) \subset \pi\left(\mathcal{P} \cap \mathcal{W}^{+} \backslash E\right)$ such that $z_{j}(\delta) \rightarrow p$ and $f\left(Q_{z_{j}(\delta)}^{c}\right) \subset Q_{w_{j}(\delta)}^{\prime}$, where $\left\{w_{j}(\delta)\right\}$ is a certain sequence converging to $w(\delta)$ and with $\left|w_{j}(\delta)\right|=\delta$. We will show that this is impossible by proving the following: 


\section{XIAOJUN HUANG}

Claim 4.3. For each $\left\{z_{j}\right\} \subset \mathcal{W}^{+} \cap \mathcal{P}$ such that $z_{j} \rightarrow p$, there is a real analytic ray $\gamma_{0} \subset Q_{p} \cap D$ with $p$ as its starting point, such that $\left.f\right|_{\gamma_{0}} \not \equiv$ const and $\lim _{j \rightarrow \infty} Q_{z_{j}}^{c} \supset \gamma_{0}$.

Assume Claim 4.3 for the moment. Then for the sequence $\left\{z_{j}(\delta)\right\}$ as chosen above, we have $f\left(\gamma_{0}\right) \subset Q_{w(\delta)}^{\prime}$. Thus, by the non constancy of $f$ along $\gamma_{0}$, it follows that there are only finitely many choices of such $Q_{w(\delta)}^{\prime}$ 's. This contradicts the finiteness assumption of $A_{w}^{\prime}$ for each small $w$. Hence, to complete the proof of Lemma 4.2, we need only to prove Claim 4.3.

Proof of Claim 4.3: By the definition of $C_{t}^{+}$and the special choice of our coordinates, we see that $M_{1}$ near $p=0$ is given by an equation of the form: $\rho=2 y_{2}+\left(2 y_{1}\right)^{2 m} g(z, \bar{z})$ with $g(0,0)>0$. Here $m$ is a positive integer. Let $w=\left(b_{1}+i \tau_{1}, b_{2}+i \tau_{2}\right) \in \mathcal{W}^{+}$with $\tau_{2}>\left|\tau_{1}\right|$. Then $Q_{w}$ is defined by the following equation: $z_{2}=b_{2}-i \tau_{2}-i(-1)^{m}\left(z_{1}-b_{1}+i \tau_{1}\right)^{2 m} g(z, \bar{w})$. For $z \in Q_{w}$,

$\rho(z, \bar{z})=2\left(-\tau_{2}+(-1)^{m+1} \operatorname{Re}\left(\left(z_{1}-b_{1}+i \tau_{1}\right)^{2 m} g(z, \bar{w})\right)\right)+\left(2 y_{1}\right)^{2 m} g(z, \bar{w})$.

Notice that for $w \in \mathcal{W}^{+}$as above, we can write $g(z, \bar{w})=l\left(b_{1}, b_{2}\right)+O\left(\tau_{2}+\right.$ $\left.\left|z-\left(b_{1}, b_{2}\right)\right|\right)$ with $l\left(b_{1}, b_{2}\right)$ greater than some fixed positive constant. Also, write $z_{1}=b_{1}-i \tau_{1}+\xi=b_{1}-i \tau_{1}+r e^{i \theta}$ with $r \geq 0$. Then, for $z \in Q_{w}$, $g(z, \bar{w})=l\left(b_{1}, b_{2}\right)+O\left(\tau_{2}+r\right)$ and

$$
\begin{aligned}
\rho(z, \bar{z}) & =-2 \tau_{2}+(-1)^{m+1} 2 \operatorname{Re} \xi^{2 m}\left(l\left(b_{1}, b_{2}\right)+O\left(\tau_{2}+r\right)\right)+ \\
& +\left(2\left(-\tau_{1}+\operatorname{Im} \xi\right)\right)^{2 m}\left(l\left(b_{1}, b_{2}\right)+O\left(\tau_{2}+r\right)\right) .
\end{aligned}
$$

Hence, $z \in Q_{w} \cap D$ if

$$
\begin{aligned}
&-2 \tau_{2}+2(-1)^{m+1} \operatorname{Re} \xi^{2 m}\left(l\left(b_{1}, b_{2}\right)+O\left(\tau_{2}+r\right)\right)+ \\
&\left(2\left(-\tau_{1}+\operatorname{Im} \xi\right)\right)^{2 m}\left(l\left(b_{1}, b_{2}\right)+O\left(\tau_{2}+r\right)\right)<0 ;
\end{aligned}
$$

or if

$$
(-1)^{m+1} 2 \operatorname{Re} \xi^{2 m}\left(1+O\left(\tau_{2}+r\right)\right)+(2(\operatorname{Im} \xi))^{2 m}\left(1+O\left(\tau_{2}+r\right)\right)<0 .
$$




\section{SCHWARZ REFLECTION PRINCIPLE}

Now, it is easy to see that the left hand side of the above inequality can be written as

$$
(-1)^{m+1} 2 r^{2 m} \cos (2 m \theta+\phi(w, z))+(1+\epsilon(w, z)) r^{2 m}(2 \sin \theta)^{2 m},
$$

where $\phi(w, z), \epsilon(w, z) \rightarrow 0$ as $r, \tau_{2} \rightarrow 0$.

When $m$ is even, the left hand side of (4.2) is not greater than $-0.5 r^{2 m}$ for $\theta=0$ and for $\tau_{2}, r$ sufficiently close to 0 . When $m>1$ is odd, we let $2 m \theta=\pi$. Then the left hand side of (4.2) can be written in the following form:

$$
\begin{gathered}
\left(-2+\left(2 \sin \frac{\pi}{2 m}\right)^{2 m}+O\left(\tau_{2}+r\right)\right) r^{2 m} \leq\left(-2+\left(\frac{\pi}{m}\right)^{2 m}+O\left(\tau_{2}+r\right)\right) r^{2 m} \leq \\
\leq\left(-2+\left(\frac{\pi}{3}\right)^{6}+O\left(\tau_{2}+r\right)\right) r^{2 m} \leq-0.4 r^{2 m},
\end{gathered}
$$

when $m \geq 3$ and when $\tau_{2}, r$ are sufficiently small.

Also notice that $\bar{w}=\left(b_{1}-i \tau_{1}, b_{2}-i \tau_{2}\right)$. Write the defining equation of $Q_{w}$ as $z_{2}=h\left(z_{1}, \bar{w}\right)$ for $w \in \mathcal{W}^{+}$with $|w|$ sufficiently small. Then the above discussion indicates that for some fixed (small) $r_{0}>0, Q_{w}^{c} \supset$ $\gamma_{w}=\left\{(r, h(r, \bar{w})): r_{0}>r \geq 0\right\}$ in case $m$ is even; and $Q_{w}^{c} \supset \gamma_{w}=$ $\left\{\left(r e^{i \frac{\pi}{2 m}}, h\left(r e^{i \frac{\pi}{2 m}}, \bar{w}\right)\right): r_{0}>r \geq 0\right\}$ in case $m$ is odd. Moreover, it is also clear that $\left(\lim _{w\left(\in \mathcal{W}^{+}\right) \rightarrow 0} Q_{w}^{c}\right) \cap D \supset \gamma_{0}=\left\{(r, h(r, 0)): r_{0} \geq r>0\right\}$ in case $m$ is even; and $\left(\lim _{w\left(\in \mathcal{W}^{+}\right) \rightarrow 0} Q_{w}^{c}\right) \cap D \supset \gamma_{0}=\left\{\left(r e^{i \frac{\pi}{2 m}}, h\left(r e^{i \frac{\pi}{2 m}}, 0\right)\right): r_{0} \geq r>0\right\}$ in case $m$ is odd.

Since $Q_{p} \backslash\{p\}$ cannot stay completely inside $D$, by the pseudoconvexity of $C_{t}^{+}$near $p=0$. Thus each connected component of $Q_{p} \cap D \cap \widetilde{\mathcal{P}}$ intersects $M_{1}$ along some one dimensional real analytic subset. On the other hand, by a simple and direct computation, one can easily verify that $\widetilde{\mathcal{P}} \cap C_{t}^{+} \cap Q_{0}=\{0\}$ after making $\widetilde{\mathcal{P}}$ small. (We would like to mention that this fact was already observed in the work of Diederich-Pinchuk [23].) Hence, by Lemma 2.2 (h) and Lemma 3.1, we see that $\left.f\right|_{\gamma_{0}}$ is not constant. The proof of Claim 4.3 is complete now.

Lemma 4.4. $f$ extends across each point in $C_{t}^{+}$.

Proof of Lemma 4.4: By Lemma 4.2, it suffices for us to show that $f$ extends almost everywhere across $C_{t}^{+}$. This can be similarly done by using 


\section{XIAOJUN HUANG}

Lemma 4.1 and the argument in Lemma 2.3, taking into account the above mentioned simple fact (first due to [23]) that $C_{t}^{+} \cap Q_{p} \cap O(p)=\{p\}$, for each $p \in C_{t}^{+}$.

\section{§5. Completion of the Proof of the Main Theorem - Assuming Theorem C}

By now, we have shown that $f$ extends holomorphically across $M_{1} \backslash C_{o}$.

Lemma 5.1. Let $p \in M_{1}$ and let $C$ be a real analytic arc in $M_{1}$. Suppose that $Q_{p} \cap \overline{\widetilde{\mathcal{P}}} \cap C=\{p\}$. Also, suppose that $f \in C^{\omega}\left(M_{1} \backslash C\right)$. Then $f$ admits a holomorphic extension across $p$.

We first assume Lemma 5.1 and see how the proof of the Main Theorem follows:

Let $C^{*}=\left\{z \in C_{o}\right.$ : either $\operatorname{dim}_{R} Q_{z} \cap C_{o}=1$ or $z$ is a singular point of $\left.C_{o}\right\}$. We first notice, by the finiteness of $A_{z}$ for each $z \in \mathcal{P}$, that $C^{*}$ is a discrete set in $M_{1}$ (see [22]). Now Lemma 5.1 indicates that $f \in C^{\omega}\left(M_{1} \backslash C^{*}\right)$. On the other hand, once we know that $f$ extends across $M_{1} \backslash C^{*}$, then for each point $p \in C^{*}$, we can easily construct an arc as in Lemma 5.1. Therefore, we see that $f$ also extends holomorphically across $C^{*}$. This completes the proof of our main Theorem assuming Theorem C.

We next present the proof of Lemma 5.1, which will be divided, for clarity, into several steps. The main idea is similar to that which appeared in the proof of Lemma 3.1.

We also would like to mention that the above lemma was obtained in the work of Diederich-Fornaess-Ye [22] in case $f$ is a CR homeomorphism.

\section{Proof of Lemma 5.1:}

STEP I: After making $\mathcal{P}$ small, we can assume that for each $z \in D^{c} \cap \mathcal{P}$, $Q_{z} \cap C$ is a finite set. Moreover, we claim that we can also assume that $Q_{z} \cap \widetilde{\mathcal{P}} \cap M_{1}$ is a one dimensional real analytic subset for each $z \in D^{c} \cap \mathcal{P}$. Hence by Lemma $2.2(\mathrm{~h})$ and the hypothesis, we conclude for each $z \in$ 


\section{SCHWARZ REFLECTION PRINCIPLE}

$\mathcal{P} \cap D^{c}$, that $f$ is not constant when restricted to each connected component of $Q_{z} \cap \widetilde{\mathcal{P}} \cap D$.

Indeed, suppose that there is a sequence $z_{j}\left(\in D^{c}\right) \rightarrow p, Q_{z_{j}} \cap \widetilde{\mathcal{P}} \cap M_{1}$ is a finite set, then $Q_{z_{j}} \cap \widetilde{\mathcal{P}} \cap D$ is connected and its boundary is contained in $Q_{z_{j}} \cap \partial \widetilde{\mathcal{P}} \cap \bar{D} \cup\{$ a finite set $\}$. (In particular, by letting $j \rightarrow \infty$, one sees that $Q_{p} \cap \bar{D} \cap \partial \widetilde{\mathcal{P}} \neq \emptyset$.) From the continuity principle, it follows that $f$ extends holomorphically across any point in $Q_{z_{j}} \cap \widetilde{\mathcal{P}} \cap \bar{D}$. Now, notice that the hypothesis indicates that $f$ extends to a fixed neighborhood of $Q_{p} \cap \partial \widetilde{\mathcal{P}} \cap \bar{D}$. Thus a standard application of the maximum principle indicates that the convergence radius of $f$ at each point in $Q_{z_{j}} \cap \widetilde{\mathcal{P}} \cap D$ is greater than the smallest convergence radius of $f$ along $Q_{z_{j}} \cap \partial \widetilde{\mathcal{P}} \cap \bar{D}$, which is greater than some positive constant for $j$ sufficiently large (see, for example, [5] for a related argument). Hence it follows, in particular, that $f$ extends holomorphically across $p=0$. Thus, we are done in this case.

STEP II: This step can be skipped if for some choice of $\widetilde{\mathcal{P}}$,

$$
\left(Q_{p} \cap \bar{D} \cap \partial \widetilde{\mathcal{P}}\right)=\emptyset .
$$

Assume that for any choice of $\widetilde{\mathcal{P}},\left(Q_{p} \cap \bar{D} \cap \partial \widetilde{\mathcal{P}}\right) \neq \emptyset$. Then as in the beginning of Lemma 4.2, there are a small $\epsilon>0$ and a finite set $\left\{p_{j}\right\}_{j=1}^{N} \subset Q_{p} \cap \bar{D} \cap \partial \widetilde{\mathcal{P}}$ such that $f$ extends to the union of the balls $B_{2 \epsilon}\left(p_{j}\right)$ with $\left(Q_{p} \cap \bar{D} \cap \partial \widetilde{\mathcal{P}}\right) \subset$ $\cup_{j} B_{\epsilon}\left(p_{j}\right)$ and $p \notin \overline{B_{2 \epsilon}\left(p_{j}\right)}$. Arguing in the same way as in Lemma 4.2 and by using the result established in Step I, we can assume that $f$ is finite to one when restricted to each $\overline{B_{\epsilon}\left(p_{j}\right)} \cap Q_{p}$. Now, it is clear that there are only finitely many $\{w\}$ 's in $\overline{\mathcal{P}^{\prime}}$ such that $f\left(Q_{p} \cap B_{\epsilon}\left(p_{l}\right)\right) \subset Q_{w}^{\prime}$ for some $l$. Hence, there are only finitely many $\xi^{\prime}$ s in $\cup_{j}\left(\overline{B_{\epsilon}\left(p_{j}\right)} \cap Q_{p}\right)$, which can be mapped to the set $\left\{\mathcal{R}^{\prime}(w)\right\}$. Therefore, after shrinking $\widetilde{\mathcal{P}}$ slightly, if necessary, we can assume, in the following, that the above mention $\xi$ 's do not meet $\partial \widetilde{\mathcal{P}}$.

STEP III: After the above preparation, we now are ready to use the idea that appeared in the proof of Lemma 3.1 to finish the proof of Lemma 5.1. First, we fix a small $\mathcal{P}^{\prime}$ and then choose a sufficiently small $\widetilde{\mathcal{P}}$ such that (i) the property in Step II holds, (ii) $\mathcal{R}^{\prime}(f(\overline{\widetilde{\mathcal{P}}} \cap \bar{D})) \subset \subset \mathcal{P}^{\prime}$. 


\section{XIAOJUN HUANG}

Still choose $S$ such that $S \cap\left(M_{1}^{s} \cup M_{1}^{a}\right)$ is dense in $S$. Pick $p_{0} \in S \cap$ $\left(M_{1}^{s} \cup M_{1}^{a}\right) \cap \operatorname{Bih}_{M_{1}}(f) \backslash \bar{E}$ which is sufficiently close to $p=0$; and write $\mathcal{V}^{p_{0}}(E)$ for the 2-dimensional component of $\mathcal{V}(E)$, which contains the graph of $f$ over $O\left(p_{0}\right) \cap \mathcal{W}^{+} \cap \mathcal{P} \backslash E$. Here $E=E_{0} \cup E^{*}$. As before, we will seek a contradiction suppose that $\pi: \mathcal{V}^{p_{0}}(E) \rightarrow \mathcal{W}^{+} \cap \mathcal{P} \backslash E$ is not surjective. Then, we can find a curve $\gamma:(0,1] \rightarrow \mathcal{W}^{+} \cap \mathcal{P} \backslash E$ such that $\gamma(0)=p_{0}$, $\gamma(1) \notin \pi\left(\mathcal{V}^{p_{0}}\right)$, and $\gamma((0,1)) \subset \pi\left(\mathcal{V}_{1}^{p_{0}}\right)$. Moreover, we can assume that there is a unique lift of $\gamma(t)$ to $\widetilde{\gamma}(t) \subset \mathcal{V}_{1}^{p_{0}}$ (for $\left.t \in(0,1)\right)$ such that $\widetilde{\gamma}(0)=\left(p_{0}, f\left(p_{0}\right)\right.$ ), $\widetilde{\gamma}(t)=(\gamma(t), w(t))$ for some continuous function $w(t)(t \in[0,1))$, and $\pi$ is locally biholomorphic at each $\widetilde{\gamma}(t)$ for $t \in(0,1)$. We have $f\left(Q_{\gamma(t)}^{c}\right) \subset Q_{w(t)}^{\prime}$ for $t \in(0,1)$. Meanwhile, for each small $t$, there is a $\xi(t) \in Q_{\gamma(t)}^{c}$ such that $f(\xi(t))=w^{*}(t)$.

Let $\delta$ be the maximal value of $\tau$ which has the following property: For each $t \in(0, \tau)$, there is a $\xi(t) \in Q_{\gamma(t)} \cap D \cap \widetilde{\mathcal{P}}$ such that $f(\xi(t))=w^{*}(t)$ and the germ $\mathcal{V}^{*}(\gamma(t))$ of the complex analytic variety, which is defined near $\widetilde{\gamma(t)}$ by the holomorphic conditions:

$$
f\left(Q_{z} \cap O(\xi(t)) \subset Q_{w}^{\prime} \text { and } f\left(Q_{z}^{c}\right) \subset Q_{w}^{\prime},\right.
$$

is of dimension 2 at $\widetilde{\gamma}(t)$.

Then $\delta>0$ by the above observation. Since $\lim _{t \rightarrow 1^{-}} Q_{\gamma(t)}^{c} \supset Q_{\gamma(1)}^{c}$, as in the proof of Lemma 3.1, we then need only to show that $\delta=1$ to get a contradiction. Indeed, if $\delta=1$, then for any limit point $w(1)$ of $w(t)$ as $t \rightarrow 1^{-}$, since $f\left(Q_{\gamma(1)}^{c}\right) \subset Q_{w(1)}^{\prime}$ and $w^{*}(1) \in f(\overline{\widetilde{\mathcal{P}}} \cap \bar{D})$, we can conclude that $(\gamma(1), w(1)) \in \mathcal{V}^{p_{0}}(E)$ and thus reach a contradiction.

Suppose that $\delta<1$. For $t<\delta$, let $\xi(t)$ be chosen as above. Then we have three cases to study. (i) $\lim _{t_{j} \rightarrow \delta^{-}} \xi(t)=\xi_{0} \in \partial \widetilde{\mathcal{P}} \backslash M_{1}$, (ii) $\lim _{t_{j} \rightarrow \delta^{-}} \xi(t)=$ $\xi_{0} \in M_{1}$; and (iii) $\lim _{t_{j} \rightarrow \delta^{-}}=\xi_{0} \in D \cap \widetilde{\mathcal{P}}$ for some subsequence $\left\{t_{j}\right\}$.

We first claim that Case (i) can be excluded if we make $\mathcal{P}$ small. Indeed, suppose that this is not the case. Then there are sequences $\left\{z_{j}\right\}$, $\left\{w_{j}\left(z_{j}\right)\right\}\left(\subset \mathcal{P}^{\prime}\right)$, and $\left\{\xi_{j}\left(z_{j}\right)\right\} \subset \partial \widetilde{\mathcal{P}} \cap \bar{D}$ such that $z_{j}\left(\in \mathcal{W}^{+} \backslash E\right) \rightarrow p$, $f\left(\xi_{j}\left(z_{j}\right) Q_{z_{j}}\right) \subset Q_{w_{j}\left(z_{j}\right)}^{\prime}$, and $f\left(\xi_{j}\left(z_{j}\right)\right)=w_{j}^{*}\left(z_{j}\right)$. By passing to the limit, we see a contradiction to our special arrangement of $\widetilde{\mathcal{P}}$ as in Step II. 


\section{SCHWARZ REFLECTION PRINCIPLE}

We next show that Case (ii) can be excluded, too. Also, suppose not. Then after taking the limit, we get $f\left(\xi_{0}\right)=w^{*}(\delta)=w(\delta) \in M_{2}$, and $f\left(Q_{\gamma(\delta)}^{c}\right) \subset$ $Q_{w(\delta)}^{\prime}$. By our choice of $E$, it follows that $w(\delta) \notin W L\left(M_{2}\right)$. Therefore, Lemma 4.1 indicates that $f$ extends holomorphically across $\xi_{0}$. In particular, we have both $f\left(Q_{\gamma(\delta)} \cap O\left(\xi_{0}\right)\right) \subset Q_{w(\delta)}^{\prime}$ and $f\left(Q_{\xi_{0}} \cap O\left(\xi_{0}\right)\right) \subset Q_{w(\delta)}^{\prime}$. On the other hand, the following claim says that $\gamma(\delta) \in A_{\xi_{0}}$. Then, an easy fact shows that $\gamma(\delta) \in M_{1}$ (see [19], for example). This gives us a contradiction.

Claim 5.2. With the above notation and assumptions, it then holds that $\gamma(\delta) \in A_{\xi_{0}}$.

Proof of Claim 5.2: Let $\rho$ and $\rho^{\prime}$ be the defining functions of $M_{1}$ and $M_{2}$ near $\xi_{0}$ and $f\left(\xi_{0}\right)(=w(\delta))$, respectively. Then we notice that $\rho^{\prime}(f(\xi), \overline{f(\eta)})=$ $\rho(\xi, \bar{\eta}) h(\xi, \bar{\eta})$ for $\xi, \eta \in \Omega$ and $h \neq 0$ by Lemma $2.2(\mathrm{~h})$. Here $\Omega$ and $\Omega^{\prime}$ are small open neighborhoods near $\xi_{0}$ and $f\left(\xi_{0}\right)$, respectively; and $f$ is proper from $\Omega$ to $\Omega^{\prime}$.

For each $\omega \in \Omega^{\prime}$, let $\left.f\right|_{\Omega} ^{-1}(\omega)=\left\{\sigma_{j}(\omega)\right\}$. For $\xi \in \cup_{j=1}^{k} \sigma_{j}\left(f\left(Q_{\xi_{0}} \cap \Omega\right)\right)$, there is a point $\widetilde{\xi} \in Q_{\xi_{0}} \cap \Omega$ such that $f(\xi)=f(\widetilde{\xi})$. Now, from $\left.\rho^{\prime}\left(f(\xi), \overline{f\left(\xi_{0}\right)}\right)\right)=$ $\rho\left(\xi, \overline{\xi_{0}}\right) h\left(\xi, \overline{\xi_{0}}\right)$ and $\rho^{\prime}\left(f(\widetilde{\xi}), \overline{f\left(\xi_{0}\right)}\right)=\rho\left(\widetilde{\xi}, \overline{\xi_{0}}\right) h\left(\widetilde{\xi}, \overline{\xi_{0}}\right)=0$, it follows that $\rho\left(\xi, \overline{\xi_{0}}\right)=0$. Thus, we conclude that $\xi \in Q_{\xi_{0}}$. On the other hand, using the properness of $f$ near $\xi_{0}$, we notice that $f\left(Q_{\xi_{0}} \cap O\left(\xi_{0}\right)\right) \supset Q_{w(\delta)}^{\prime} \cap O(w(\delta))$. The hypothesis in Claim 5.2 then indicates that $Q_{\gamma(\delta)} \cap O\left(\xi_{0}\right) \subset \cup_{j} \sigma_{j}\left(f\left(Q_{\xi_{0}} \cap\right.\right.$ $\left.O\left(\xi_{0}\right)\right)$ ). We thus conclude that $Q_{\gamma(\delta)}$ and $Q_{\xi_{0}}$ have a piece near $O\left(\xi_{0}\right)$ in common. Therefore, we see that $Q_{\xi_{0}} \cap \widetilde{\mathcal{P}}=Q_{\gamma(\delta)} \cap \widetilde{\mathcal{P}}$. That is, $\gamma(\delta) \in A_{\xi_{0}}$. The proof of Claim 5.2 is complete.

Hence, Case (iii) is the only possibility to study. Now, by passing to the limit, we conclude that $f\left(Q_{\gamma(\delta)} \cap O\left(\xi_{0}\right)\right) \subset Q_{w(\delta)}^{\prime}$ with $f\left(\xi_{0}\right)=w^{*}(\delta)$.

Still, we let $\mathcal{V}^{*}(\widetilde{\gamma}(\delta))$ be the germ of the complex analytic variety near $(\gamma(\delta), w(\delta))$ which is defined by the holomorphic conditions $f\left(Q_{z} \cap O\left(\xi_{0}\right)\right) \subset$ $Q_{w}^{\prime}$ and $f\left(Q_{z}^{c}\right) \subset Q_{w}^{\prime}$. We notice that it is contained in the germ of $\mathcal{V}^{p_{0}}(E)$ at $\widetilde{\gamma}(\delta)$. Also, by using a similar fact as in Lemma 2.2 (c), one sees that $\mathcal{V}^{*}(\widetilde{\gamma}(\delta))$ must be of dimension 2 at $\widetilde{\gamma}(\delta)$.

On the other hand, we assumed that $\mathcal{V}^{p_{0}}$ is smooth at $\widetilde{\gamma}(\delta)$. It thus 


\section{XIAOJUN HUANG}

follows that $\mathcal{V}^{*}(\widetilde{\gamma}(\delta))$ is the same as the germ of $\mathcal{V}^{p_{0}}$ at $\widetilde{\gamma}(\delta)$. Thus, for $z \approx \gamma(\delta)$ and $(z, w) \in \mathcal{V}^{p_{0}} \cap O(\widetilde{\gamma}(\delta)), f\left(Q_{z} \cap O\left(\xi_{0}\right)\right) \subset Q_{w}^{\prime}$. By Step I, we notice that $f$ is not constant along $Q_{z} \cap O\left(\xi_{0}\right)$. As in Lemma 3.1, one can then apply the Hurwitz theorem to conclude that for $z$ sufficiently close to $\gamma(\delta), w^{*}(z) \in f\left(Q_{z} \cap O\left(\xi_{0}\right)\right)$, where $(z, w(z)) \in O(\gamma(\delta)) \cap \mathcal{V}^{p_{0}}$. This clearly contradicts the maximality of our $\delta$.

Finally, the proof of Lemma 5.1 is complete.

\section{Part II: Regularity of Holomorphic Correspondences -Proof of Theorem C}

\section{$\S 6$. Statement of Theorem D and Related Notation}

Part II of this paper is devoted to the proof of Theorem C stated in $\S 2$, which has been one of the key ingredients in the proof of our Main Theorem. In fact, Theorem $\mathrm{C}$ will be a special case of the following Theorem D.

In this part, except in $\S 11$, we always let $M_{1}$ and $M_{2}$ be two connected hypersurfaces of finite D'Angelo type in $\mathbf{C}^{n+1}$, and let $f$ be a non-constant continuous $\mathrm{CR}$ mapping from $M_{1}$ into $M_{2}$, which maps $p=0 \in M_{1}$ to $q=0 \in M_{2}$. Assume that $f$ extends holomorphically to the side $D$ of $M_{1}$.

We will always choose normal coordinates for $M_{2}$ near $q=0$ in what follows.

Let $\rho_{2}$ and $\widetilde{\rho_{2}}$ be the normalized defining equations of $M_{2}$ near 0 , as introduced in (1.1) and (1.2), respectively. Then, for example, $\widetilde{\rho_{2}}$ takes the following form:

$$
\widetilde{\rho_{2}}(w, \bar{w})=w_{n+1}+\overline{w_{n+1}}+\sum_{j=0}^{\infty} \widetilde{\psi_{j}}\left(w^{\prime}, \overline{w^{\prime}}\right)\left(w_{n+1}\right)^{j},
$$

where $\widetilde{\psi}_{j}$ contain no harmonic terms and $w^{\prime}=\left(w_{1}, \cdots, w_{n}\right)$.

Similar to the notion introduced in [3] and [1], we define the reflection function $G(f, \lambda)$ as follows:

$$
G(f(z), \lambda)=-f_{n+1}(z)-\sum_{j=0}^{\infty} \widetilde{\psi_{j}}\left(f^{*}(z), \lambda\right)\left(f_{n+1}(z)\right)^{j},
$$




\section{SCHWARZ REFLECTION PRINCIPLE}

where $f^{*}=\left(f_{1}, \cdots, f_{n}\right)$.

Also, after a holomorphic change of variables, we let $S=\mathbf{R}^{n+1} \cap O(0) \subset$ $M_{1}$ be a totally real submanifold containing 0 . Still write $\mathcal{W}^{ \pm}$for the standard wedges with edge $S$ near 0 , similar to what was introduced in $\S 2$. We recall the notion of the holomorphic correspondence extension of $f$ to $\left(\mathcal{W}^{+} \cap \mathcal{P} ; \mathcal{P}^{\prime}\right)$, as defined in Definition 2.1 in the 2 dimensional case. That is, $f$ extends as a holomorphic correspondence to $\left(\mathcal{W}^{+} \cap \mathcal{P} ; \mathcal{P}^{\prime}\right)$ if there is a negligible set $E \subset \mathcal{W}^{+} \cap \mathcal{P}$ and a complex analytic variety $\mathcal{V} \subset\left(\mathcal{W}^{+} \cap \mathcal{P} \backslash E\right) \times \mathcal{P}^{\prime}$ of dimension $n+1$ such that the first natural projection $\pi$ is finite to one, and is surjective when restricted to each irreducible component of dimension $n+1$. Moreover $\mathcal{V}$ contains the graph of $f$ over $\mathcal{W}^{+} \cap \mathcal{P} \cap O\left(p_{j}\right) \backslash E$, where $\left\{p_{j}\right\}$ is a certain sequence converging to 0 and $p_{j} \in(S \backslash \bar{E}) \cap \operatorname{Bih}_{M_{1}}(f)$.

Write $\mathcal{V}^{(n+1)}(E)$ for the union of all $n+1$ dimensional irreducible components. Then $\pi$ will be an analytic cover from $\mathcal{V}^{(n+1)}$ to $\mathcal{W}^{+} \cap \mathcal{P} \backslash E$, by the above assumption. For each $z \in \mathcal{W}^{+} \cap \mathcal{P} \backslash E$, write $\pi^{\prime}\left(\left.\pi\right|_{\mathcal{V}^{n+1}(E)} ^{-1}(z)\right)=$ $\left\{w^{(1)}(z), \cdots, w^{(N)}(z)\right\}$, where $\pi^{\prime}$ is the natural projection of $\mathcal{V}$ to $\mathcal{P}^{\prime}$. Here $N$ is independent of $z$. Then any symmetric function of $\left\{w^{(1)}(z), \cdots, w^{(N)}(z)\right\}$ is holomorphic over $\mathcal{W}^{+} \cap \mathcal{P} \backslash E$.

Part II is devoted to proving the following result, whose last statement is the content of Theorem C:

Theorem D. Let $M_{1}, M_{2}, S, \mathcal{W}^{ \pm}, f$, and $p=q=f(p)=0$, be as above. Assume that $f$ admits a holomorphic extension across an open dense subset of $M_{1}$. Also, assume that for sufficiently small $\mathcal{P}$ and $\mathcal{P}^{\prime}, f$ extends as a holomorphic correspondence to $\left(\mathcal{W}^{+} \cap \mathcal{P} ; \mathcal{P}^{\prime}\right)$. Then

(a) $G(f(z), \lambda)$ extends as a holomorphic function to $O_{z}(0) \times O_{\lambda}(0)$.

(b) After shrinking $D$ suitably, $f$ extends as a proper holomorphic mapping from $D$ to its image.

(c) In case $n=1, f$ admits a holomorphic extension across $p=0$.

We next describe the main ideas for the proof of Theorem D: The proof of Theorem D (a) will be crucial to the whole argument, which is based on the weak version of the edge of the wedge theorem. This assertion essentially tells that $f$ extends as a multi-valued map $\mathcal{F}$ across $M_{1}$ near $p=0$. The 


\section{XIAOJUN HUANG}

next major step toward the proof of Theorem D is to connect the branching locus of $\mathcal{F}$ with the Segre varieties of $M_{1}$ and $M_{2}$. After achieving these, we can obtain the Baouendi-Rothschild Hopf lemma in our setting, from which Theorem D (b) follows easily. Finally, all these facts, together with an application of the Hartogs extension theorem, complete the proof of Theorem $\mathrm{D}$ (c), i.e, the proof of Theorem C.

\section{$\S 7$ : Extension of $G(f, \lambda)$ - Proof of Theorem D (a)}

From now on, we always keep the assumption in the statement of Theorem D. Also we keep the previously established notation. In this section, we prove the following lemma, which is the content of Theorem D (a).

Lemma 7.1. Under the above notation and assumptions, $G(f(z), \lambda)$ extends holomorphically to $O_{z}(0) \times O_{\lambda}(0)$.

The proof we present here is to take the differentiation along the boundary. Comparing to the study of smooth CR-mappings as in [31], [34], [3], [1], etc, there is an essential difference here. That is, our map is not assumed to be smooth. So, we can only do it almost everywhere. To reach the bad points, we jump into the domain and use the hypothesis to control the rate of blowing-up so that we can apply the edge of the wedge theorem (see also Chapter 2 of [28]).

Proof of Lemma 7.1: First, we notice that $G(f(z), \lambda)$ is holomorphic over $D \times O_{\lambda}(0)$ and continuous over $\left(D \cup M_{1}\right) \times O_{\lambda}(0)$. By the definition of $G(f(z), \lambda)$ and using the assumption that $f\left(M_{1}\right) \subset M_{2}$, we have

$$
\overline{f_{n+1}(z)}=G\left(f(z), \overline{f^{*}(z)}\right) \text { for } z \in M_{1} \text {. }
$$

Shrinking the size of $M_{1}$ if necessary, we can choose a basis $\left\{\mathcal{L}_{j}\right\}_{j=1}^{n}$ for the complex tangent subbundle $\mathrm{T}^{(1,0)} M_{1}$ of $M_{1}$, whose coefficients are real analytic in $z$. Applying $\overline{\mathcal{L}}_{j}$ to (7.1), we obtain

$$
\overline{\mathcal{L}}_{j} \overline{f_{n+1}(z)}=\sum_{l=1}^{n} \frac{\partial G}{\partial \lambda_{l}} \overline{\mathcal{L}}_{j} \bar{f}_{l}, \quad \text { for } z \in \operatorname{Bih}_{M_{1}}(f)
$$




\section{SCHWARZ REFLECTION PRINCIPLE}

Here the notation $\operatorname{Bih}_{M_{1}}(f)$ is the same as before . By our non-constancy assumption of $f$ and a result in [8], $\operatorname{Bih}_{M_{1}}(f)$ is a dense open subset in $M_{1}$. Let $J=\operatorname{det}\left(\overline{\mathcal{L}}_{j} \bar{f}_{l}\right)_{1 \leq j, l \leq n}$ and let $\mathcal{J}$ be the matrix $\left(\overline{\mathcal{L}}_{j} \bar{f}_{l}\right)_{1 \leq j, l \leq n}$, which are well-defined over $\operatorname{Bih}_{M_{1}}(f)$. Then, we claim that $J \neq 0$ on $\operatorname{Bih}\left(M_{1}\right)$. Indeed, for each $z \in \operatorname{Bih}\left(M_{1}\right)$, the rank of the vectors $\left\{\mathcal{J}_{1}(z), \cdots, \mathcal{J}_{n}(z)\right\}$ is $n$, where $\mathcal{J}_{j}(z)=\left(\mathcal{L}_{j} f_{1}, \cdots, \mathcal{L}_{j} f_{n}, \mathcal{L}_{j} f_{n+1}\right)$. Write $\widetilde{\mathcal{J}}_{j}(z)=\left(\mathcal{L}_{j} f_{1}, \cdots, \mathcal{L}_{j} f_{n}\right)$. Then, (7.2) indicates that the rank of $\left\{\widetilde{\mathcal{J}}_{1}(z), \cdots, \widetilde{\mathcal{J}}_{n}(z)\right\}$ is also $n$. Hence, $J(z) \neq 0$.

For $z \in \operatorname{Bih}\left(M_{1}\right)$, we now have

$$
\left(\frac{\partial G}{\partial \lambda_{1}}, \cdots, \frac{\partial G}{\partial \lambda_{n}}\right)^{t}=\mathcal{J}^{-1}(z, \bar{z}, \overline{D f})\left(\overline{\mathcal{L}}_{1} \bar{f}_{n+1}, \cdots, \overline{\mathcal{L}}_{n} \bar{f}_{n+1}\right)^{t} .
$$

Writing (7.3) as $n$ scalar equations, applying $\overline{\mathcal{L}}_{j}$ to each of them, and proceeding in this manner, we see, by induction, that for each multi-index $\alpha=\left(\alpha_{1}, \cdots, \alpha_{n}\right)$, there are two holomorphic functions $g_{\alpha}^{(1)}$ and $g_{\alpha}^{(2)}$ in the arguments $\left(z, \bar{z}, \bar{f}, \cdots, \overline{D^{k} f}, \cdots, \overline{D^{|\alpha|} f}\right)$ ( where $\left.k \leq|\alpha|\right)$ ) such that for each $z \in \operatorname{Bih}\left(M_{1}\right)$, one has $g_{\alpha}^{(2)}\left(z, \bar{z}, \cdots, \overline{D^{|\alpha|} f}\right) \neq 0$ and

$$
D_{\lambda}^{\alpha} G\left(f, \overline{f^{*}(z)}\right)=\frac{g_{\alpha}^{(1)}\left(z, \bar{z}, \cdots, \overline{D^{|\alpha|} f}\right)}{g_{\alpha}^{(2)}\left(z, \bar{z}, \cdots, \overline{D^{|\alpha|} f}\right)} .
$$

Here $D^{k}$ denotes the vector formed by all derivatives of $f$ with order $k$. We remark that $g_{\alpha}^{(j)}$ is actually a polynomial in $\left(\overline{D f}, \cdots, \overline{D^{|\alpha|} f}\right)(j=1,2)$ with coefficients real analytic in $z$ for $z$ close to $M_{1}$. By passing to the limit, we see that the function $\frac{g_{\alpha}^{(1)}}{g_{\alpha}^{(2)}}$ has a continuous extension to $M_{1}$, which we will denote by $h_{\alpha}\left(z, \bar{z}, \cdots, \overline{D^{|\alpha|} f}\right)$. Notice also that for $\|w\|,\|\lambda\| \ll 1$, there exists a large constant $R$ so that $\left|D_{\lambda}^{\alpha} G(w, \lambda)\right| \lesssim \alpha ! R^{|\alpha|}$.

Denote by $\eta_{j, l}(z)(j=1, \cdots, n+1, l=1, \cdots, N)$ the $j^{\text {th }}$-component of $w^{(l)}(z)$, where $\pi^{\prime}\left(\left.\pi\right|_{\mathcal{V}^{n+1}(E)} ^{-1}(z)\right)=\left\{w^{(1)}(z), \cdots, w^{N}(z)\right\}$.

Consider the following equation in $X$ :

$$
\begin{gathered}
\prod_{l_{1}, \cdots, l_{n+1}}\left(X-\frac{1}{\alpha !}\left(D_{\lambda}^{\alpha} G\left(\eta_{1, l_{1}}(z), \cdots, \eta_{n+1, l_{n+1}}(z), \overline{f_{1}(\bar{z})}, \cdots, \overline{f_{n}(\bar{z})}\right)\right)\right. \\
=X^{N^{*}}+\sum_{j<N^{*}} c_{j}(z) X^{j}=0
\end{gathered}
$$




\section{XIAOJUN HUANG}

where $l_{j}$ runs from 1 to $N$ for each $j \leq n+1$, and $N^{*}=N^{n+1} \cdot c_{j}(z)^{\prime}$ s can be seen to be the symmetric functions of $w^{(j)}(z)^{\prime}$ 's and hence can be seen to be holomorphic and bounded over $O(0) \cap \mathcal{W}^{+} \backslash E$. Moreover, one can obtain the Cauchy estimates $\left|c_{j}(z)\right| \lesssim R^{N^{*}|\alpha|}$. In particular, each $c_{j}(z)$ has a limit up to $\mathbf{R}^{n+1} \cap O(0)$ in the distribution sense (see [3], for example). Now, one can easily verify that

$$
\widetilde{h}_{\alpha}(z)=\frac{1}{\alpha !} h_{\alpha}\left(z, z, \cdots, \overline{D^{|\alpha|} f(\bar{z})}\right)
$$

satisfies the above equation when $z$ is in a certain open subset of $\mathbf{R}^{n+1}$ near 0 . Meanwhile, $\widetilde{h}_{\alpha}(z)$ clearly extends to a meromorphic function to $\mathcal{W}^{+} \cap O_{z}(0)$; for $f$ is holomorphic over $\mathcal{W}^{-}(\subset D)$. Thus, by the uniqueness of holomorphic functions (see for example, [33]), it follows that

$$
\left(\widetilde{h}_{\alpha}(z)\right)^{N^{*}}+\sum_{j<N^{*}} c_{j}(z)\left(\widetilde{h}_{\alpha}(z)\right)^{j}=0
$$

for

$$
z \in \mathcal{W}^{+} \cap O_{z}(0) \backslash\left(\left\{\text { the singular set of } \widetilde{h_{\alpha}} \text { in } \mathcal{W}^{+} \cap O_{z}(0)\right\} \cup E\right) .
$$

In particular, we see that $\widetilde{h}_{\alpha}(z)$ is bounded. Using the Riemann extension theorem, we conclude that $\widetilde{h_{\alpha}}$ extends holomorphically to $\mathcal{W}^{+} \cap O_{z}(0)$. Moreover, we have $\left|\widetilde{h}_{\alpha}(z)\right| \lesssim R^{N^{*}|\alpha|}$ for $z \in \mathcal{W}^{+} \cap O_{z}(0)$; for its coefficients have the same sort of estimates. Also, since they have boundary values which are continuous over $S$, we see that $\widetilde{h}_{\alpha}$ extends continuously to $\mathcal{W}^{+} \cup S$.

Next, fix a small open subset $U$ containing 0 . Let

$$
\phi_{\alpha}^{+}(z)=\frac{1}{\alpha !}\left(D_{\lambda}^{\alpha} \sum_{\beta} \widetilde{h}_{\beta}(z)\left(\lambda-\overline{f^{*}(\bar{z})}\right)^{\beta}\right)_{\lambda=0}
$$

for $z \in \mathcal{W}^{+} \cap U$ and let $\phi_{\alpha}^{-}(z)=\left.\frac{1}{\alpha !} D_{\lambda}^{\alpha} G(f(z), \lambda)\right|_{\lambda=0}$ for $z \in \mathcal{W}^{-} \cap U$.

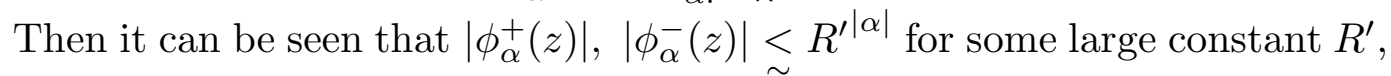
where $z$ stays in their defining regions, respectively. Notice that $\phi_{\alpha}^{+}$matches 


\section{SCHWARZ REFLECTION PRINCIPLE}

up with $\phi_{\alpha}^{-}$over $U \cap \mathbf{R}^{n+1}$. The classic edge of the wedge theorem then indicates that $\phi_{\alpha}^{-}$extends to a holomorphic function $\phi_{\alpha}$ defined over some sufficiently small neighborhood $U^{\prime}$ of 0 , whose size depends only on the size of the wedges where $\phi_{\alpha}^{ \pm}$are defined, and therefore is independent of $\alpha$ (see [37] or [4], for example). Moreover, $U^{\prime}$ can be filled in by analytic disks with boundary staying in the closure of $\left(\mathcal{W}^{+} \cup \mathcal{W}^{-}\right) \cap U([37])$. So, the maximal principle tells that $\phi_{\alpha}$ has the same kind of Cauchy estimates as $\phi_{\alpha}^{+}$and $\phi_{\alpha}^{-}$ do. Now, $\left(\sum_{\alpha} \phi_{\alpha} \lambda^{\alpha}\right)$ clearly gives the holomorphic extension for $G(f(z), \lambda)$ to $O_{z}(0) \times O_{\lambda}(0)$. The proof of Lemma 7.1 is now complete.

As an immediate application of Lemma 7.1, we let $\lambda=0$ and conclude that $f_{n+1}$ admits a holomorphic extension to a neighborhood of 0 . Also, by the Nullstellensatz (see [6]), one sees from the holomorphic property of $G(f, \lambda)$, that each $f_{j}$ satisfies an irreducible polynomial equation, with leading coefficient 1 and other coefficients holomorphic near 0.

In what follows, we denote, by $\mathcal{V} \subset O_{z}(0) \times O_{w}(0) \subset \mathbf{C}^{n+1} \times \mathbf{C}^{n+1}$, the irreducible analytic variety of dimension $n+1$, which contains the graph of $f$ over $D$ near $p=0$ and is extended to a neighborhood of the origin by the above mentioned equations annihilating $f_{j}$ 's. Use $\pi$ and $\pi^{\prime}$ for the natural projections to the first and second copies of $\mathbf{C}^{n+1}$, respectively. Write $\mathcal{F}(z)=\pi^{\prime}\left(\pi^{-1}(z)\right)$, which can be regarded as the multiple-valued extension of $\left.f\right|_{D}$ to a neighborhood $U^{(0)}$ of $0 \in \mathbf{C}^{n+1}$. Clearly, $\mathcal{F}(0)=\{0\}$ and, near the origin, $\lim _{z_{j} \rightarrow z} \mathcal{F}\left(z_{j}\right)=\mathcal{F}(z)$ and $G(\mathcal{F}(z), \lambda)$ is single valued.

We notice that $\mathcal{V}$ is an analytic cover over $O_{z}(0)$. Write $\mathcal{E}$ for the union of the branch locus of the irreducible polynomial equation annihilating $f_{j}$ $(j=1, \cdots, n)$. Near the origin, $\pi$ is then a covering mapping from $\mathcal{V} \backslash \pi^{-1}(\mathcal{E})$ to its image.

Write $(\mathcal{Y}, \sigma, \mathcal{V})$ for the standard normalization of $\mathcal{V}$ (see [40], Chapter 8). We remark that after making $\mathcal{V}$ small, $\sigma^{-1}(0)$ is a single point. Write $\mathcal{E}_{0}=\pi \circ \sigma(\operatorname{Sing})$, where

Sing $=\left\{x \in \mathcal{Y}\right.$ : either $\mathrm{x}$ is singular or $\mathrm{x}$ is smooth but $\mathrm{d}_{x} \sigma$ is singular $\}$.

Write $\operatorname{Sing}_{0}=(\pi \circ \sigma)^{-1}(\pi \circ \sigma)(\operatorname{Sing})$. Then $\pi \circ \sigma$ is a local biholomorphic 


\section{XIAOJUN HUANG}

mapping from $\mathcal{Y} \backslash$ Sing $_{0}$ to its image and gives a finitely sheeted covering mapping away from the singular set. (see [40], Chapter 8 and [27], pp 108). Therefore, if $\mathcal{V}$ is singular at 0 , i.e, $f$ is not holomorphic at 0 , then $\mathcal{E}_{0}=$ $\pi \circ \sigma($ Sing $)$ is a non-trivial analytic variety. We call $\mathcal{E}_{0}$ the genuine branch locus of $\mathcal{F}$ or $\mathcal{V}$. By the basic fact concerning branched covering spaces, it is known that $\mathcal{E}_{0}$ is of codimension 1 everywhere. Also, it is easy to see that for each $z \in \mathcal{E}_{0}$, there is a sequence $\left\{z_{j}\right\} \rightarrow z$ such that $\# \pi^{-1}(z)<\# \pi^{-1}\left(z_{j}\right)$ for each $j$. Moreover, for each $z \notin \mathcal{E}_{0}, \mathcal{F}$ can be split into several holomorphic branches near $z$.

\section{§8. Branches of $\mathcal{F}$ and Segre Varieties}

We now start to study the connection between $\mathcal{F}$ and the Segre varieties of $M_{1}$ and $M_{2}$. Our basic tool will be the reflection function introduced in Theorem D (a). We first prove the following

Lemma 8.1. After shrinking $\mathcal{P}$, if necessary, then for each $z \in \mathcal{P}$, it holds that $\mathcal{F}(z) \subset A_{\widetilde{f(z)}}^{\prime}$ and $\mathcal{F}\left(Q_{z}\right) \subset Q_{\widetilde{f(z)}}^{\prime}$ for any $\widetilde{f(z)} \in \mathcal{F}(z)$.

Proof of Lemma 8.1: We mention that by a simple unique continuation argument and by using the invariant property of Segre varieties, one can easily show that for each "nice" branch $\widetilde{f}$ with $\left.\widetilde{f}\right|_{D}=f$, it holds that $\widetilde{f}\left(Q_{z}\right) \subset$ $Q_{\widehat{f(z)}}^{\prime}$. The main idea of the proof of the lemma is to use the fact that $\widetilde{\rho}_{2}\left(\mathcal{F}(z), \widetilde{f}^{*}(\omega)\right)$ is single-valued for each fixed $\omega$, by the above established reflection function.

We let $z \in \mathcal{P} \cap D$, and assume that $z, z^{*} \notin \mathcal{E}$. Here, as before, we use $z^{*}$ to denote the reflection point of $z$, i.e, $z^{*}=\mathcal{R}(z)$. We also choose a simply connected smooth curve $\gamma:[0,1] \rightarrow U^{(0)}$ with $\gamma \subset \mathcal{P} \backslash D \backslash \mathcal{E}, \gamma(1)=z^{*}$, and $\gamma \cap M_{1}=\{\gamma(0)\}$. Here, when there is no confusion arising, we also use the letter $\gamma$ to denote its image set. Moreover, we assume that $\gamma$ intersects $M_{1}$ transversally at $\gamma(0)$. Let $\widetilde{\gamma}=\mathcal{R}(\gamma) \cup \gamma$. Then $\widetilde{\gamma}$ is still a simply connected curve in $\widetilde{\mathcal{P}}$. Thickening $\widetilde{\gamma}$ suitably, we can then obtain a simply connected domain, which we will denote by $O(\subset \widetilde{\mathcal{P}})$. Now, we can define a holomorphic 


\section{SCHWARZ REFLECTION PRINCIPLE}

map $\hat{f}$ from $O$, which coincides with $f$ on $O \cap D$. For a subset $A$, write $\operatorname{conj}(A)=\{\bar{z}: z \in A\}$. Let $M_{c}=\left\{(z, \omega) \in U^{(0)} \times \operatorname{conj}\left(U^{(0)}\right): \rho_{1}(z, \omega)=0\right\}$ and consider $M_{c}^{*}=M_{c} \cap\{O \times \operatorname{conj}(O)\}$, where $\rho_{1}$ is the defining equation of $M_{1}$ near 0 . Now, by Lemma 7.1, the following function is well-defined and holomorphic over $M_{c}^{*}$ :

$$
\Xi(z, \omega)=\overline{f_{n+1}(\bar{\omega})}-G\left(\mathcal{F}(z), \overline{\hat{f}^{*}(\bar{\omega})}\right)\left(=\widetilde{\rho_{2}}\left(\mathcal{F}(z), \overline{\hat{f}^{*}(\bar{\omega})}\right)\right)
$$

which vanishes on $M^{*}=\left\{(a, \bar{a}): a \in M_{1} \cap U^{(0)}\right\}$. Notice that $M^{*}$ is a totally real subset in $M_{c}^{*}$ of maximal dimension, we conclude from the uniqueness property of holomorphic functions [33] that $\Xi(z, \omega) \equiv 0$ in the union, denoted by $M_{c}^{* *}$, of the connected components of $M_{c}^{*}$ which have nonempty intersections with $M^{*}$. By our choice of $\widetilde{\gamma}$, we see that $\left(z, \overline{z^{*}}\right),\left(z^{*}, \bar{z}\right) \in$ $M_{c}^{* *}$; for $\left.(\gamma(t), \overline{\mathcal{R}(\gamma(t)})\right)\left(\in M_{c}^{*}\right)(\mathcal{R}(\gamma(t)), \overline{\gamma(t)})\left(\in M_{1 c}^{*}\right)(t \in[0,1])$ connect them to $M^{*}$, respectively. Therefore,

$$
\left(z, \operatorname{Conj}\left(Q_{z} \cap O\left(z^{*}\right)\right)\right) \subset M_{c}^{*},\left(z^{*}, \operatorname{Conj}\left(Q_{z^{*}} \cap O(z)\right)\right) \subset M_{c}^{*}
$$

by the definition of Segre varieties. This implies that $G\left(\mathcal{F}(z), \overline{\hat{f}^{*}(\omega)}\right)=$ $\overline{f_{n+1}(\omega)}$ for $\omega \in Q_{z} \cap O\left(z^{*}\right)$; and $G\left(\mathcal{F}\left(z^{*}\right), \overline{\hat{f}^{*}(\omega)}\right)=\overline{f_{n+1}(\omega)}$ for $\omega \in Q_{z^{*}} \cap$ $O(z)$. Equivalently, we have $\widetilde{\rho}_{2}\left(\mathcal{F}(z), \overline{\hat{f}^{*}(\omega)}\right)=0$ for $\omega \in Q_{z} \cap O\left(z^{*}\right)$; and $\widetilde{\rho}_{2}\left(\mathcal{F}\left(z^{*}\right), \overline{\hat{f}^{*}(\omega)}\right)=0$ for $\omega \in Q_{z^{*}} \cap O(z)$. From a basic fact of Segre varieties: $w \in Q_{z}$ if and only if $z \in Q_{w}$; it now follows that $\hat{f}\left(Q_{z} \cap O\left(z^{*}\right)\right) \subset \cap Q_{\widehat{f(z)}}^{\prime}$ with $\widetilde{f}(z) \in \mathcal{F}(z)$; and $\hat{f}\left(Q_{z^{*}} \cap O(z)\right) \subset \cap Q_{\widetilde{f}\left(z^{*}\right)}^{\prime}$ with $\widetilde{f}\left(z^{*}\right) \in \mathcal{F}\left(z^{*}\right)$.

By slightly perturbing $z$ if necessary, we assume momentarily that $J_{\hat{f}} \neq 0$ near $z^{*}$. Now, since $\hat{f}\left(Q_{z} \cap O\left(z^{*}\right)\right) \subset Q_{\tilde{f}(z)}^{\prime}$ for any $\widetilde{f}(z) \in \mathcal{F}(z)$, and since each of them is a connected complex submanifold of dimension $n$ near $\hat{f}\left(z^{*}\right)$, all these submanifolds therefore coincide near $\hat{f}\left(z^{*}\right)$. Hence, it follows easily that all $Q_{\widetilde{f}(z)}^{\prime} \cap \widetilde{\mathcal{P}}$ are the same. So, $\mathcal{F}(z) \subset A_{\tilde{f}(z)}^{\prime}$ for any given $\widetilde{f}(z) \in \mathcal{F}(z)$.

Assume also that $J_{f}(z) \neq 0$. In a similar manner, we then also see that $\mathcal{F}\left(z^{*}\right) \subset A_{\tilde{f}\left(z^{*}\right)}^{\prime}$ for any given $\widetilde{f}\left(z^{*}\right) \in \mathcal{F}\left(z^{*}\right)$.

Next, we note that $\widetilde{\rho}_{2}\left(\mathcal{F}(z), \overline{f^{*}}(\omega)\right)=0$ if and only if $\rho_{2}(\mathcal{F}(z), \overline{\hat{f}}(\omega))=$ 0 . Let $q=\left(q^{\prime}, q_{n+1}\right) \in \mathcal{F}(z)$. Then, by what we just obtained and by 


\section{XIAOJUN HUANG}

the reality of $\rho_{2}$, we have $\rho_{2}(\hat{f}(\omega), \bar{q})=0$ and therefore, $\widetilde{\rho_{2}}\left(\hat{f}(\omega), \overline{q^{\prime}}\right)=0$ for $\omega \in Q_{z} \cap O\left(z^{*}\right)$. Now, since Lemma 7.1 indicates that $\widetilde{\rho_{2}}\left(\mathcal{F}(\omega), \overline{q^{\prime}}\right)$ is well defined and holomorphic for $\omega \in U^{(0)}$, we conclude, in particular, that $\widetilde{\rho_{2}}\left(\mathcal{F}(\omega), \overline{q^{\prime}}\right)=0$ for $\omega \in \widetilde{\mathcal{P}} \cap Q_{z}$. This implies that $\mathcal{F}\left(Q_{z}\right) \subset Q_{q}^{\prime}$.

In a similar manner, we can also show that $\mathcal{F}\left(Q_{z^{*}}\right) \subset Q_{q^{*}}^{\prime}$ for any $q^{*} \in$ $\mathcal{F}\left(z^{*}\right)$.

Finally, we can complete the proof of Lemma 8.1 by passing to the limit.

Let $z$ be close to 0 such that either $z \in \mathcal{E} \cup \mathcal{R}(\mathcal{E}) \cup M_{1}$, or $J_{f}(z)=0$, or $J_{f}\left(z^{*}\right)=0$. By what we did above, we can find a sequence $\left\{z_{j}\right\}$ with $z_{j} \rightarrow z$ so that $\mathcal{F}\left(z_{j}\right) \subset A_{\tilde{f}\left(z_{j}\right)}^{\prime}$ for any $\widetilde{f}\left(z_{j}\right) \in \mathcal{F}\left(z_{j}\right)$. In the other words, $Q_{\tilde{f}\left(z_{j}\right)}^{\prime}=$ $Q_{\hat{f}\left(z_{j}\right)}^{\prime}$ for any $\widetilde{f}\left(z_{j}\right), \hat{f}\left(z_{j}\right) \in \mathcal{F}\left(z_{j}\right)$. It then follows that $Q_{\tilde{f}(z)}^{\prime}=Q_{\hat{f}(z)}^{\prime}$ for any $\widetilde{f}(z), \hat{f}(z) \in \mathcal{F}(z)$, i.e, $\mathcal{F}(z) \in Q_{\widetilde{f}(z)}^{\prime}$ for any $\widetilde{f}(z) \in \mathcal{F}(z)$. Similarly, we also have $\mathcal{F}\left(Q_{z}\right) \subset Q_{\tilde{f}(z)}^{\prime}$ for any $\widetilde{f}(z) \in \mathcal{F}(z)$.

Hence, after shrinking $\mathcal{P}$ one more time if necessary, we see the proof of Lemma 8.1.

Remark 8.2 (a) By Lemma 8.1, we can now define $Q_{\mathcal{F}(z)}^{\prime}$ to be $Q_{q}^{\prime}$ and $A_{\mathcal{F}(z)}^{\prime}=A_{q}^{\prime}$ for some $q \in \mathcal{F}(z)$. Then Lemma 8.1 can be written as $\mathcal{F}(z) \subset A_{\mathcal{F}(z)}^{\prime}$ and $\mathcal{F}\left(Q_{z}\right) \subset Q_{\mathcal{F}(z)}^{\prime}$.

(b) As an application of Lemma 8.1, we conclude that when $A_{w}^{\prime}$ is just a single point for $w \approx 0$, then $f$ extends holomorphically near 0 . This is the case when the target point is a Levi non-degenerate point or has some special bi-type property. Define $\mathcal{A}^{\prime}$ by sending each point $w$ to $A_{w}^{\prime}$. One can see that if $f$ does not allow holomorphic extension, then $\mathcal{A}^{\prime}$ branches at 0 (we will make this more precise later). It is this fact that links the branch points of $\mathcal{F}$ with the singular points of $\mathcal{A}^{\prime}$, which will be one the key observations for the proof of Theorem D (c).

For example, let $D_{1}=\left\{\left(z_{1}, z_{2}\right):\left|z_{1}\right|^{2}+\left|z_{2}\right|^{2}<1\right\}$ and $D_{2}=\left\{\left(z_{1}, z_{2}\right)\right.$ : $\left.\left|z_{1}\right|^{4}+\left|z_{2}\right|^{2}<1\right\}$. Let $\mathcal{G}=\left(\sqrt{z_{1}}, z_{2}\right)$ be the multiple-valued map from $D_{1}$ to $D_{2}$. Then the branch locus of $\mathcal{G}$ is given by $Z=\left\{\left(0, z_{2}\right)\right\}$, and $\mathcal{G}(Z)=\left\{\left(0, z_{2}\right)\right\}$. We observe that $\mathcal{G}(Z)$ is exactly the branch locus of the $\mathcal{A}^{\prime}$-map of $\partial D_{2}$. 


\section{SCHWARZ REFLECTION PRINCIPLE}

Lemma 8.3. (i) Let $M_{2}$ be given in the normal coordinates with a normalized real-valued defining equation $\rho_{2}$ (see (1.1)). Let $\Omega^{ \pm}$be defined by $\pm \rho_{2}<0$ respectively. Write $\hat{n}^{ \pm}=\left\{b=\left(0^{\prime}, b_{n+1}\right): \pm b_{n+1}<0\right\}$ with $\left|b_{n+1}\right|$ small. For $b \in \hat{n}^{+}\left(b \in \hat{n}^{-}\right.$, respectively) then $A_{b}^{\prime} \subset \Omega^{+},\left(A_{b}^{\prime} \subset \Omega^{-}\right.$, respectively).

(ii) If for some $q \in \mathcal{F}(z)$, it holds that $q \in M_{2}$, then $\mathcal{F}(z) \subset M_{2}$. Hence, $\mathcal{F}\left(M_{1} \cap \mathcal{P}\right) \subset M_{2}$.

(iii) $f_{n+1}(z)=z_{n+1}^{k} g(z)$ for some holomorphic function $g(z)$ defined near 0 and for some positive integer $k$.

(iv) After shrinking $\widetilde{\mathcal{P}}$, it holds that $\left\{\mathcal{F}^{-1}(0)\right\} \cap \widetilde{\mathcal{P}}=\{0\}$, i.e, $\widetilde{f}(z) \neq 0$ for any $z(\in \widetilde{\mathcal{P}}) \neq 0$ and for any $\widetilde{f}(z) \in \mathcal{F}(z)$. Moreover $\mathcal{F}^{-1}(w) \cap \widetilde{\mathcal{P}}$ is a finite set for any $w \approx 0$; and for any analytic variety $V$ passing through $0 \in \mathcal{P}, \mathcal{F}(V)$ also gives the germ of an analytic variety at $0 \in \mathcal{P}^{\prime}$ with $\operatorname{dim}_{0} V=\operatorname{dim}_{0}(\mathcal{F}(V))$. (v) After shrinking $\mathcal{P}$ if necessary, then for any $z \in \mathcal{P}$, either $\mathcal{F}(z) \subset \Omega^{+}$, or $\mathcal{F}(z) \subset \Omega^{-}$, or $\mathcal{F}(z) \subset M_{2}$.

Proof of Lemma 8.3: (i). Let $b=\left(0, b_{n+1}\right)$ be as in Part (i) of Lemma 8.3. Let $\eta=\left(\eta^{\prime}, \eta_{n+1}\right) \in A_{b}^{\prime}$, i.e, $Q_{\eta}^{\prime} \cap \widetilde{\mathcal{P}}^{\prime}=Q_{b}^{\prime} \cap \widetilde{\mathcal{P}}^{\prime}$. We see that

$$
\begin{aligned}
\left\{\left(w^{\prime}, w_{n+1}\right)\right. & \left.\in \widetilde{\mathcal{P}}^{\prime}: w_{n+1}+\overline{\eta_{n+1}}+\rho_{2}^{*}\left(w^{\prime}, \bar{\eta}^{\prime}, \frac{w_{n+1}-\overline{\eta_{n+1}}}{2 i}\right)=0\right\} \\
& =\left\{\left(w^{\prime}, w_{n+1}\right) \in \widetilde{\mathcal{P}}^{\prime}: w_{n+1}+b_{n+1}=0\right\}
\end{aligned}
$$

where $\rho_{2}^{*}=\sum_{j \geq 0} \psi_{j}\left(w^{\prime}, \overline{w^{\prime}}\right)\left(\operatorname{Im} w_{n+1}\right)^{j}$. Letting $w^{\prime}=0$, we see that $\eta_{n+1}=$ $b_{n+1}$. Meanwhile, since $\left\{\left(w^{\prime},-\eta_{n+1}\right): w^{\prime} \in \mathbf{C}^{n}\right\} \cap \widetilde{\mathcal{P}}^{\prime}=Q_{\eta}^{\prime}$, it follows that $\rho_{2}^{*}\left(w^{\prime}, \overline{\eta^{\prime}}, i \eta_{n+1}\right)=0$ for $w^{\prime}$ with $\left(w^{\prime}, b_{n+1}\right) \in \widetilde{\mathcal{P}}^{\prime}$. We see, in particular, that $\rho_{2}^{*}\left(\eta^{\prime}, \overline{\eta^{\prime}}, i \eta_{n+1}\right)=0$. Therefore, $\psi_{0}\left(\eta^{\prime}, \overline{\eta^{\prime}}\right)=-\sum_{j>0}^{\infty} \psi_{j}\left(\eta^{\prime}, \overline{\eta^{\prime}}\right)\left(i \eta_{n+1}\right)^{j}=$ $O\left(\left|\eta^{\prime}\right|^{2}\left|\eta_{n+1}\right|\right)$. Now, $\rho_{2}(\eta, \bar{\eta})=2 \eta_{n+1}+\rho_{2}^{*}\left(\eta^{\prime}, \overline{\eta^{\prime}}, 0\right)=2 \eta_{n+1}+\psi_{0}\left(\eta^{\prime}, \overline{\eta^{\prime}}\right)+$ $O\left(\left|\eta^{\prime}\right|^{2}\left|\eta_{n+1}\right|\right)=2 \eta_{n+1}+O\left(\left|\eta^{\prime}\right|^{2} \eta_{n+1}\right)$, which is positive when $b_{n+1}=\eta_{n+1}$ is positive and small; and is negative when $b_{n+1}<0$. This gives the proof of part (i).

(ii). Let $q \in M_{2} \cap \mathcal{F}(z)$. Then $q \in Q_{q}^{\prime}=Q_{\widetilde{f}(z)}^{\prime}$ for each $\widetilde{f}(z) \in \mathcal{F}(z)$. Thus $\widetilde{f}(z) \in Q_{q}^{\prime}=Q_{\widetilde{f}(z)}^{\prime}$, by Lemma 8.1. So, $\widetilde{f(z)} \in M_{2}$ (see [19]). 


\section{XIAOJUN HUANG}

(iii). By Lemma 8.1, it follows that $\mathcal{F}\left(Q_{0}\right) \subset Q_{\mathcal{F}(0)}^{\prime}=Q_{0}^{\prime}$. Thus, we see that $f_{n+1}\left(z^{\prime}, 0\right) \equiv 0$. Since $f_{n+1}$ is holomorphic near 0 , we conclude that $f_{n+1}\left(z^{\prime}, z_{n+1}\right)=z_{n+1}^{k} g(z)$ for some holomorphic function $g$ near 0 and some positive integer $k$.

(iv). Let $\mathcal{V}$ be as at the end of $\S 7$. In the following discussion, we let $U$ be such that $\widetilde{\mathcal{P}} \supset U \supset \mathcal{P}$ and restrict $\pi$ to $\pi^{-1}(U)$.

Notice that it holds that $\mathcal{F}^{-1}(0) \cap U=\pi\left(\pi^{\prime-1}(0) \cap \pi^{-1}(U)\right)$. Thus, if 0 is an accumulation point of $Y=\mathcal{F}^{-1}(0)$, then $Y \cap U$ is an analytic variety of $U$ and it would have positive dimension at 0 . We will assume this and seek a contradiction.

Then, $Y \cap U$ contains some holomorphic curve $Y^{*} \subset \mathcal{P}$ parametrized by $z^{\prime}=\phi(t), z_{n+1}=\psi(t),\left(t \in \Delta\right.$, the unit disk in $\left.\mathbf{C}^{1}\right)$ with $\phi(0)=\psi(0)=0$, $\|d \phi\|+\|d \psi\| \neq 0$ for $t \neq 0$. For each $z \in Y \cap \mathcal{P}, \mathcal{F}\left(Q_{z}\right) \subset Q_{0}^{\prime}$, by Lemma 8.1. We claim that $\cup Q_{z}$ with $z \in Y^{*}$ fills in an open subset in $\mathbf{C}^{n+1}$. This then gives us a contradiction; for we assumed that $J_{f} \not \equiv 0$ and $Q_{0}^{\prime}$ is a complex hypersurface.

To see the size of $U^{*}=\cup Q_{z}$ with $z \in Y^{*}$, we choose the normal coordinates for $M_{1}$ near $p=0$ with the normalized defining equation $\overline{\widetilde{\rho}_{1}(z, \bar{z})}=0$ as in (1.2). Therefore, it can be seen that $Q_{z}$ can be parametrized by

$$
z_{n+1}=-\overline{\psi(t)}-\sum_{j=0}^{\infty} \overline{\widetilde{\phi}_{j}}\left(\overline{\phi(t)}, z^{\prime}\right) \overline{\psi(t)}^{j} \equiv g\left(z^{\prime}, \bar{t}\right)
$$

Here and in what follows, we use the notation $\bar{h}(z)$ for the function $\overline{h(\bar{z})}$. So, $U^{*}$ can be parametrized by the map

$$
\mathcal{T}\left(z^{\prime}, \bar{t}\right): \mathbf{C}^{n} \times \Delta \rightarrow \mathbf{C}^{n+1}
$$

$\left.\mathcal{T}\left(z^{\prime}, \bar{t}\right)=\left(z^{\prime},-\overline{\psi(t)}-\sum_{j=0}^{\infty}{\widetilde{\widetilde{\phi}_{j}}}_{(\overline{\phi(t)}}, z^{\prime}\right) \overline{\psi(t)}{ }^{j}\right)$. To see that $\mathcal{T}\left(z^{\prime}, t\right)$ is a biholomorphism at a certain point $\left(z^{\prime}, \bar{t}\right)(\approx(0,0))$, it suffices to show that $\frac{\partial g}{\partial \bar{t}} \neq 0$ at $\left(z^{\prime}, \bar{t}\right)$. Indeed, this can be argued as follows: When $\psi(t) \not \equiv 0$, we can simply choose $\left(0^{\prime}, t\right)$ for some $t$ with $\psi^{\prime}(t) \neq 0$; when $\psi \equiv 0$, if $\frac{\partial g}{\partial \bar{t}} \equiv 0$, then for any given $z^{\prime}$ with $\left|z^{\prime}\right|$ small, $\overline{\widetilde{\phi_{0}}}\left(\overline{\phi(t)}, z^{\prime}\right)$ is independent of $t$. Hence 


\section{SCHWARZ REFLECTION PRINCIPLE}

it has to be 0 ; for $\phi(0)=0$ and thus $\overline{\phi_{0}}\left(\phi(0), z^{\prime}\right)=0$. Letting $z^{\prime}=\phi(t)$, it follows that $\overline{\widetilde{\phi_{0}}}(\overline{\phi(t)}, \phi(t)) \equiv 0$. This contradicts the finite type assumption on $M_{1}$; for it implies that $Y^{*}=(\phi(t), 0)$ stays inside $M_{1}$. So, by making $\widetilde{\mathcal{P}}$ small, it holds that $\mathcal{F}^{-1}(0) \cap \widetilde{\mathcal{P}}=\{0\}$.

Therefore, we conclude that 0 is an isolated point of $\pi^{\prime-1}(0)$. Hence, $\pi^{\prime}$ is locally finite near 0 . Moreover, $\mathcal{F}^{-1}$, when restricted as a map from $O(0)$ to $\widetilde{\mathcal{P}}$, maps a small neighborhood $0 \in \widetilde{\mathcal{P}}^{\prime}$ into a small neighborhood of $0 \in \mathcal{P}$.

Now, shrinking $\widetilde{\mathcal{P}}$ if necessary, we assume that $q \approx 0, \mathcal{F}^{-1}(q) \cap \widetilde{\mathcal{P}}=$ $\pi\left(\pi^{\prime-1}(q)\right)$ is finite. By the elimination theorem ([16], Theorem 1 of pp122), there exists an open neighborhood $U^{\prime}$ of 0 such that $\pi^{\prime}$ is proper from $\pi^{\prime-1}\left(U^{\prime}\right)$ to $U^{\prime}$; and away from a proper analytic set, $\pi^{\prime}$ gives a sheeted covering map. In particular, $\pi^{\prime}$ is an open mapping.

In the following discussion, we make $\mathcal{F}(U) \subset U^{\prime}$, and we restrict $\pi^{\prime}$ to $\pi^{\prime-1}\left(U^{\prime}\right)$. By the above discussion, we also observe the fact: $\pi^{-1}(U) \subset$ $\pi^{\prime-1}\left(U^{\prime}\right)$.

Using again the elimination theorem and noting that $\pi, \pi^{\prime}$ are local analytic covering maps, it follows that for any analytic variety $V \subset U, \mathcal{F}(V) \cap$ $\pi^{\prime}\left(\pi^{-1}(U)\right)=\pi^{\prime}\left(\pi^{-1}(V) \cap\left(\pi^{-1}(U)\right)\right)$ is an analytic variety in $\pi^{\prime}\left(\pi^{-1}(U)\right)$ with the same dimension at the origin (see for example, Theorem $11 \mathrm{E} \mathrm{pp}$ 68 of [40]; or Theorem 1, pp122, of [16]). This completes the proof of (iv).

(v) Since $\pi$ is proper, it is also closed. Also, both are open mappings; for they are local analytic covering mappings, too.

For any closed subset $B$ of $U^{\prime}$, we first notice that

$$
\mathcal{F}^{-1}(B) \cap U=\pi\left(\pi^{\prime-1}(B) \cap \pi^{-1}(U)\right) .
$$

Since $\pi^{\prime-1}(B)$ is closed in $\pi^{\prime-1}(B) \cap \pi^{\prime-1}\left(U^{\prime}\right)$, it is closed in $\pi^{\prime-1}(B) \cap \pi^{-1}(U)$ by the above arrangement. By using the fact that $\pi$ is closed, it follows that $\mathcal{F}^{-1}(B)$ is closed in $U$. In particular, we see that $\mathcal{F}^{-1}\left(M_{2} \cap U^{\prime}\right)$, denoted by $\widetilde{M}_{1}$, is closed in $U$. Clearly, $\mathcal{F}\left(\widetilde{M}_{1}\right) \subset M_{2}$ by part (ii) of this lemma.

Now, let $U^{0}=U \backslash \widetilde{M}_{1}$. Then it is open. We notice that $\partial U^{0}$ is contained in $\partial U \cup \widetilde{M}_{1}$. Since $\mathcal{F}=\pi^{\prime} \circ \pi^{-1}$, the openness of $\pi^{\prime}$ implies the openness of $\mathcal{F}$ from $U$ as a multiple-valued map. Therefore it sends interior points to 


\section{XIAOJUN HUANG}

interior points. On the other hand, using the continuity of $\mathcal{F}$, one sees that $\mathcal{F}$ maps the closure of $U^{0}$ to the closure of $\mathcal{F}\left(U^{0}\right)$. Hence, a simple topological argument shows that the boundary of $\mathcal{F}\left(U^{0}\right)$ is contained in $\mathcal{F}(\partial U) \cup M_{2}$. Since $\mathcal{F}^{-1}(0) \cap \widetilde{\mathcal{P}}=\{0\}$, it follows easily that 0 is not contained in the closure of $\mathcal{F}(\partial U)$. Hence, we see that there is a small ball, denoted by $B_{0}$, centered at the origin such that $B_{0} \cap \mathcal{F}(\partial U)=\emptyset$. Let $U^{*}$ be a small neighborhood of 0 such that $\mathcal{F}\left(U^{*}\right) \subset \subset B_{0}$. If $U^{0} \cap U^{*}=\emptyset$, then Part (ii) of this lemma shows that $\mathcal{F}\left(U^{*}\right) \subset \mathcal{F}\left(\widetilde{M}_{1}\right) \subset M_{2}$ and thus we are done. (In this case, $f$ must be constant). Therefore, without loss of generality, we assume that $U^{*} \cap U^{0} \neq \emptyset$. Let $D^{*}$ be an arbitrarily given connected component of $U^{0}$ with $U^{*} \cap D^{*} \neq \emptyset$. Note that $\partial \mathcal{F}\left(D^{*}\right) \subset \mathcal{F}(\partial U) \cup M_{2}$ and $\partial \mathcal{F}\left(D^{*}\right) \cap\left(B_{0} \backslash M_{2}\right)=\emptyset$. Then a simple topological argument indicates that either $\mathcal{F}\left(D^{*}\right) \supset B_{0} \cap \Omega^{+}$ or $\mathcal{F}\left(D^{*}\right) \supset \Omega^{-} \cap B_{0}$ Without loss of generality, we assume the first case. Then, there exists a point $p_{0} \in D^{*}$ and a certain $\widetilde{f}\left(p_{0}\right) \in \mathcal{F}\left(p_{0}\right)$ such that $\widetilde{f}\left(p_{0}\right) \in \hat{n}^{+}$and $\tilde{f}\left(p_{0}\right)$ is close to 0 . By the first part of this lemma and Lemma 8.1, we see that $\mathcal{F}\left(p_{0}\right) \subset \Omega^{+}$. Now, for any $z \in D^{*} \backslash \mathcal{E}$, choose a curve $\gamma$ in $D^{*}$ with $\gamma(0)=p_{0}, \gamma(1)=z$, and $\gamma((0,1)) \cap \mathcal{E}=\emptyset$. Then for any $\widetilde{f}(z) \in \mathcal{F}(z)$, there is a branch $\hat{f}$ of $\mathcal{F}$, which is continuous on $\gamma$ and $\hat{f}(z)$ coincides with $\widetilde{f}(z)$. Now, since $\hat{f} \circ \gamma$ does not meet $M_{2}$ by our choice of $U^{0}$, and $\hat{f} \circ \gamma(t) \in \Omega^{+}$for $t$ close enough to 0 (this can be seen by the fact that all limit points of $\hat{f}(\gamma(t))(t \rightarrow 0)$ are in $\left.\mathcal{F}\left(p_{0}\right)\right)$, we conclude that $\hat{f}(z)$ and thus $\tilde{f}(z)$ have to be in $\Omega^{+}$. For $z \in \mathcal{E} \cap D^{*}$, by passing to a limit and noting $\mathcal{F}(z) \cap M_{2}=\emptyset$, we can also see that $\mathcal{F}(z) \subset \Omega^{+}$. Thus, by the arbitrary choice of $D^{*}$, we see that the proof of the last part of Lemma 8.3 is complete if we make $\mathcal{P}$ small.

\section{$\S 9$. Branch Locus of $\mathcal{F}$ and Segre Varieties}

We now present some connections between the 'genuine' branch locus $\mathcal{E}_{0}$ and the Segre varieties of $M_{1}$ and $M_{2}$.

In Remark (8.2), we already noticed the importance of the points where the counting number of $A_{w}^{\prime}$ collapses. In the following, we make this more precise: 


\section{SCHWARZ REFLECTION PRINCIPLE}

As before, denote by $\mathcal{A}$ the map, which sends: $w \in \mathcal{P}$ to the finite set $A_{w} . w_{0}$ is called a separable point of $\mathcal{A}$ if $A_{w_{0}}=\left\{w_{j}\right\}_{j=0}^{N^{*}}$ satisfies the following property: There exist open neighborhoods $O_{w_{j}}$ of $w_{j}\left(j=0, \cdots, N^{*}\right)$ such that $\mathcal{A}(w) \cap O_{w_{j}}=\{w\}$ for any $w \in O_{w_{j}}$. We write $\mathcal{B}=\{w \in \mathcal{P}$ : $w$ is not a separable point of $\mathcal{A}\}$.

Similarly, we can define $\mathcal{A}^{\prime}$ and $\mathcal{B}^{\prime}$.

We will see in the following lemmas that $\mathcal{B}$ and $\mathcal{B}^{\prime}$ can be used to control the branch locus of $\mathcal{F}$.

Lemma 9.1. Let $M_{1}, M_{2}$ be as before. Then $Q_{0} \cap \mathcal{B}\left(Q_{0}^{\prime} \cap \mathcal{B}^{\prime}\right.$, respectively) is contained in a proper analytic subvariety of $Q_{0}\left(Q_{0}^{\prime}\right.$, respectively).

Proof of Lemma 9.1: We choose the normal coordinates for $M_{1}$ near $p=0$. For our purpose here, we can assume that $M_{1}$ is defined by an equation of the form:

$$
z_{n+1}+\overline{z_{n+1}}+\sum_{|\alpha|,|\beta|>0} a_{\alpha \beta} z^{\prime \alpha} \overline{z^{\prime \beta}}+\sum_{|\alpha|,|\beta| ; k>0} a_{\alpha \beta ; k} z^{\prime \alpha} \overline{z^{\prime \beta}} \overline{z_{n+1}^{k}}=0
$$

Let $P_{\alpha}\left(z^{\prime}, z_{n+1}\right)=\sum_{\beta} \overline{a_{\alpha \beta}} z^{\prime \beta}+\sum_{\beta, k} \overline{a_{\alpha \beta ; k}} z^{\prime \beta} z_{n+1}^{k}$. Then for $a=\left(a^{\prime}, a_{n+1}\right)$ $b=\left(b^{\prime}, b_{n+1}\right)$ close to the origin, $Q_{a}=Q_{b}$ if and only if $a_{n+1}=b_{n+1}$ and $P_{\alpha}\left(a^{\prime}, a_{n+1}\right)=P_{\alpha}\left(b^{\prime}, b_{n+1}\right)$. By the finite D'Angelo type assumption, it follows that the common zero of $P_{\alpha}\left(z^{\prime}, 0\right)^{\prime}$ s is 0 near the origin. Using the Noetherian property, we see that for some finitely many indices $\left\{\alpha_{j}\right\}_{j=1}^{m}$, the locus of $\left\{P_{\alpha_{j}}\left(z^{\prime}, 0\right)\right\}_{j=1}^{m}$ is also zero. Now, for a small neighborhood $U$ of 0 , define $\Lambda$ from a small neighborhood $U$ to $\mathbf{C}^{m+1}$ by $\Lambda(z)=$ $\left(z_{n+1},\left\{P_{\alpha_{j}}\left(z^{\prime}, z_{n+1}\right)\right\}_{j=1}^{m}\right)$. Then $\Lambda$ is finite to one and proper from $U$, after suitably shrinking of $U$. So, by the Remmert theorem, we conclude that the set

$$
V=\Lambda^{-1}(\Lambda(\{z \in U: \mathrm{d} \Lambda \text { does not have maximal rank at } z\}))
$$

is a proper variety of $U$ (see [19] for a similar argument). Obviously, $V \supset \mathcal{B}$. Since the projection from $V$ to the $\left\{\left(z_{1}, \cdots, z_{n}\right)\right\}$-subspace is one to one, after a linear change of coordinates in the $\left(z_{1}, \cdots, z_{n}\right)$-space, we see that $V$ can be 


\section{XIAOJUN HUANG}

defined by an equation of the form: $z_{1}^{N^{*}}+\sum_{j<N^{*}} c_{j}\left(z_{2}, \cdots, z_{n}, z_{n+1}\right) z_{1}^{j}=0$ with $c_{j}(0)=0$. In particular, it follows that $\mathcal{B} \cap Q_{0}$ is contained in a subvariety defined by $z_{1}^{N^{*}}+\sum_{j<N^{*}} c_{j}\left(z_{2}, \cdots, z_{n}, 0\right) z_{1}^{j}=0$.

Similar arguments also apply to $M_{2}$. This completes the proof of Lemma 9.1 .

Lemma 9.2. Let $M_{1}, M_{2}$, and $\mathcal{E}_{0}$ still be as before.

(a) $\mathcal{F}\left(\mathcal{E}_{0} \cap O(0)\right) \subset \mathcal{B}^{\prime}$.

(b) $\mathcal{E}_{0} \cap Q_{0} \cap O(0)$ is a proper analytic subvariety of $O(0) \cap Q_{0}$.

Proof of Lemma 9.2: (a). Let $z \in \mathcal{E}_{0}$ be sufficiently close to 0 . Then, by the definition of $\mathcal{E}_{0}$ and the discussions at the end of $\S 7$, it follows that there is a sequence $z_{j} \rightarrow z$ such that we can find two sequences $\left\{\eta_{j}\right\}$ and $\left\{\xi_{j}\right\}$ with $\xi_{j}, \eta_{j} \in \mathcal{F}\left(z_{j}\right) \eta_{j} \neq \xi_{j}$, but $\xi_{j}, \eta_{j} \rightarrow w_{0}$ for some $w_{0}$. By Lemma 8.1, it then follows that $\xi_{j} \in A_{\eta_{j}}^{\prime}$. Hence, $w_{0}$ is not a separable point of $\mathcal{A}^{\prime}$. That is, $\mathcal{F}(z) \subset \mathcal{B}^{\prime}$.

(b). By (a) and Lemma 8.1, we have $\mathcal{F}\left(Q_{0} \cap \mathcal{E}_{0}\right) \subset \mathcal{B}^{\prime} \cap Q_{0}^{\prime}$. Thus, using Lemma 8.3 (iv) and Lemma 9.1, we see the proof of the assertion in this part.

\section{§10. A Preservation Principle for $\mathcal{F}$-Proof of Theorem D (b)}

Before proceeding further, we need to strengthen Lemma 8.3 (iii) to the following version:

Lemma 10.1. $f_{n+1}(z)=z_{n+1} g(z)$ with $g(0) \neq 0$.

This sort of the Hopf lemma was established in [7] in the case when the map is assumed to be smooth. Since we now have a nice control of the branches of $\mathcal{F}$ and we know that $f_{n+1}$ is holomorphic, Lemma 10.1 can be proved by using the same approach and ideas as in [7].

Proof of Lemma 10.1: Since we use the same approach appeared in [7], we will be brief for those arguments which can be obtained from (§2, [7]). 


\section{SCHWARZ REFLECTION PRINCIPLE}

Seeking a contradiction, we suppose that $f_{n+1}(z)=z_{n+1}^{k} g(z)$ with $k \geq 2$. We start with the function $G(\mathcal{F}, \lambda)$, which has the following property:

$$
\overline{f_{2}(\omega)}=G\left(\mathcal{F}(z), \overline{\widetilde{f}^{*}(\omega)}\right)
$$

for any $z \in Q_{\omega}$ and $\left(\tilde{f}^{*}, f_{n+1}\right) \in \mathcal{F}$. Write the defining equation of $M_{1}$ in the following form (see (1.1)): $t=\phi\left(z^{\prime}, \overline{z^{\prime}}, s\right)$ with $t=2 \operatorname{Re} z_{n+1}, s=\operatorname{Im} z_{n+1}$, where $\phi\left(0, \overline{z^{\prime}}, s\right)=\phi\left(z^{\prime}, 0, s\right) \equiv 0$. Thus $z \in Q_{\omega}$ reads as

$$
z_{n+1}+\overline{\omega_{n+1}}=\phi\left(z^{\prime}, \overline{\omega^{\prime}}, \frac{z_{n+1}-\overline{\omega_{n+1}}}{2 i}\right),
$$

where, as usual, we write $\omega=\left(\omega^{\prime}, \omega_{n+1}\right)$. For $\xi \in \mathbf{C}^{n}$, write $\tau=-i s+$ $\frac{1}{2} \phi\left(z^{\prime}, \xi, s\right)$ and $R\left(z^{\prime}, \xi, \tau\right)=i s+\frac{1}{2} \phi\left(z^{\prime}, \xi, s\right)$. As in [7], using the implicit function theorem, we can find a holomorphic function $\mu\left(z^{\prime}, \xi\right)$ near 0 such that $R\left(z^{\prime}, \xi, \mu\left(z^{\prime}, \xi\right)\right) \equiv 0$ and $\mu\left(z^{\prime}, 0\right)=\mu(0, \xi) \equiv 0$. It is easy to verify that $\mu\left(z^{\prime}, \xi\right) \not \equiv 0$, for, otherwise, it implies that $\phi\left(z^{\prime}, \overline{z^{\prime}}, 0\right) \equiv 0$ and thus contradicts the finite type assumption of $M_{1}$. Now, one can directly verify that $\left(z^{\prime}, R\left(z^{\prime}, \xi, \tau\right)\right) \in Q_{\overline{(\xi, \tau)}}$. So, we have $\overline{(\xi, \tau)} \in Q_{\left(z^{\prime}, R\left(z^{\prime}, \xi, \tau\right)\right)}$ and thus

$$
\overline{f_{n+1}\left(z^{\prime}, R\left(z^{\prime}, \xi, \tau\right)\right)}=G\left(\mathcal{F}(\bar{\xi}, \bar{\tau}), \overline{\tilde{f}^{*}\left(z^{\prime}, R\left(z^{\prime}, \xi, \tau\right)\right.}\right) \text {. }
$$

As in the previous section, we write $\overline{\widetilde{f} *}(z)=\overline{\widetilde{f} *(\bar{z})}$ and use the same notation for $\overline{f_{n+1}}(z)$. Then

$$
\overline{f_{n+1}}\left(\overline{z^{\prime}}, \overline{R\left(z^{\prime}, \xi, \tau\right)}\right)=G\left(\mathcal{F}(\bar{\xi}, \bar{\tau}), \overline{\tilde{f}^{*}}\left(\overline{z^{\prime}}, \overline{R\left(z^{\prime}, \xi, \tau\right)}\right) .\right.
$$

By Lemma 9.2, we have $\mathcal{E}_{0}^{*}=\mathcal{E}_{0} \cap Q_{0}=\mathcal{E}_{0} \cap\left\{z: z_{n+1}=0\right\}$ is a local complex analytic variety of codimension at least 1 .

Now, for each non zero $z^{\prime} \notin \mathcal{E}_{0}^{*}$ which is close to the origin, there is a small neighborhood $O\left(\left(z^{\prime}, 0\right)\right)$ near $\left(z^{\prime}, 0\right)$ such that we can stratify $\mathcal{F}$ into several holomorphic branches. Next, we choose $\xi$ sufficiently small and then let $\tau$ be sufficiently close to $\mu\left(z^{\prime}, \xi\right)$. Then $R\left(z^{\prime}, \xi, \tau\right) \approx 0$, too. After letting $\widetilde{f}$ be a holomorphic branch of $\mathcal{F}$ over $O\left(\left(z^{\prime}, 0\right)\right)$, for each $l<n+1$, we then can take the derivative with respect to $\bar{z}_{l}$ in (10.1) to obtain

$$
\frac{\partial \overline{f_{n+1}}}{\partial z_{l}}+\frac{\partial \overline{f_{n+1}}}{\partial z_{n+1}} \overline{R_{z_{l}}^{\prime}\left(z^{\prime}, \xi, \tau\right)}=\sum_{j=1, \cdots, n} \frac{\partial G}{\partial \lambda_{j}}\left(\frac{\partial \overline{\widetilde{f}_{j}}}{\partial z_{l}}+\frac{\partial \overline{\widetilde{f}_{j}}}{\partial z_{n+1}} \overline{R_{z_{l}}^{\prime}\left(z^{\prime}, \xi, \tau\right)}\right)
$$




\section{XIAOJUN HUANG}

Let $\tau=\mu\left(z^{\prime}, \xi\right)$ in $(10.2)$ and notice that $R\left(z^{\prime}, \xi, \mu\left(z^{\prime}, \xi\right)\right)=0, f_{n+1}=z_{n+1}^{k} g$ with $k \geq 2$. We obtain

$$
\sum_{j=1, \cdots, n} \frac{\partial G}{\partial \lambda_{j}}\left(\frac{\partial \overline{\widetilde{f}_{j}}}{\partial z_{l}}\left(\overline{z^{\prime}}, 0\right)+\frac{\partial \overline{\widetilde{f}_{j}}}{\partial z_{n+1}}\left(z^{\prime}, 0\right) \overline{R_{z_{l}}^{\prime}\left(z^{\prime}, \xi, \tau\right)}\right)=0 .
$$

Since $\mathcal{F}$ is a finite-to-finite map, $f^{*}\left(z^{\prime}, 0\right)$ is a finite-to-one map from $O\left(z^{\prime}, 0\right) \cap$ $Q_{0}$, too. Moving to a nearby point if necessary, we can assume that

$$
\operatorname{det}\left(\frac{\partial \widetilde{\widetilde{f}}_{j}}{\partial z_{l}}\left(\overline{z^{\prime}}, 0\right)\right)_{1 \leq j, l \leq n} \neq 0 .
$$

Therefore, for each $z^{\prime} \notin \mathcal{E}_{0}^{*}$ with $\left|z^{\prime}\right|$ small, when $\xi$ is chosen so that $|\xi|$ is sufficiently smaller than $\left|z^{\prime}\right|$, using the fact that $R_{z_{l}}^{\prime}\left(z^{\prime}, 0, \tau\right)=0$ for $l<n+1$, we see that $R_{z_{l}}^{\prime}\left(z^{\prime}, \xi, \mu\left(z^{\prime}, \xi\right)\right) \approx 0$. Hence, we have for each $l<n+1$

$$
\frac{\partial G}{\partial \lambda_{l}}\left(\mathcal{F}\left(\bar{\xi}, \overline{\mu\left(z^{\prime}, \xi\right)}\right), \overline{\tilde{f}^{*}}\left(\overline{z^{\prime}}, 0\right)\right) \equiv 0 .
$$

On the other hand, letting $\tau=\mu\left(z^{\prime}, \xi\right)$ in (10.1), we have

$$
G\left(\mathcal{F}\left(\bar{\xi}, \overline{\mu\left(z^{\prime}, \xi\right)}\right), \widetilde{\widetilde{f} *}\left(\bar{z}^{\prime}, 0\right)\right) \equiv 0 .
$$

Now, as in [7], let $q_{l}=G_{\lambda_{l}}^{\prime}\left(w^{\prime}, 0, \lambda\right)$ and $q_{0}=G\left(w^{\prime}, 0, \lambda\right)$. Notice that

$$
G\left(w^{\prime}, w_{n+1}, \lambda\right)=-w_{n+1}+q_{0}-\sum_{j>0} \widetilde{\psi}_{j}\left(w^{\prime}, \lambda\right) w_{n+1}^{j}
$$

and $q_{l}=\left(q_{0}\right)_{\lambda_{l}}^{\prime}$. We see that from the equations $G\left(w^{\prime}, w_{n+1}, \lambda\right)=0$ and $G_{\lambda_{l}}^{\prime}\left(w^{\prime}, w_{n+1}, \lambda\right)=0$, we can get

$$
\left.w_{n+1}=q_{0} h^{*}\left(q_{0}, w^{\prime}, \lambda\right)\right), \quad q_{l}=q_{0} \psi_{l}^{*}\left(w^{\prime}, \lambda, q_{0}\right)
$$

for certain holomorphic functions $h^{*}, \psi_{l}^{*}$, where $l \leq n$.

In the following discussions, we always let $\tau=\mu\left(z^{\prime}, \xi\right)$. Let $w^{\prime}\left(z^{\prime}, \xi\right)=$ $\hat{f}^{*}(\bar{\xi}, \bar{\tau})$ with $\hat{f}^{*}(\bar{\xi}, \bar{\tau}) \in \mathcal{F}(\bar{\xi}, \bar{\tau})$, and let $\lambda\left(z^{\prime}\right)=\widetilde{\tilde{f}^{*}}\left(\bar{z}^{\prime}, 0\right)$ in the above formulas. We obtain the following equality

$$
q_{l}\left(\hat{f}^{*}(\bar{\xi}, \bar{\tau}), \overline{\widetilde{f}}_{l}\left(\bar{z}^{\prime}, 0\right)\right)=q_{0}\left(\hat{f}^{*}(\bar{\xi}, \bar{\tau}), \overline{\widetilde{f}^{*}}\left(\bar{z}^{\prime}, 0\right)\right) \times \psi_{l}^{*}\left(\hat{f}^{*}(\bar{\xi}, \bar{\tau}), \overline{\widetilde{f}^{*}}\left(\bar{z}^{\prime}, 0\right), q_{0}\right) .
$$




\section{SCHWARZ REFLECTION PRINCIPLE}

Here for clarity, we summarize the situations in which (10.4) makes sense and holds:

(a) $z^{\prime} \notin \mathcal{E}_{0}^{*}$ is any given point of $Q_{0}$ near the origin. (b) $\tilde{f}$ is some holomorphic branch of $\mathcal{F}$ over some small neighborhood $O\left(\left(z^{\prime}, 0\right)\right)$ of $\left(z^{\prime}, 0\right)$, whose size depends on $z^{\prime}$. (c) $\xi$ is taken in a small neighborhood $O\left(z^{\prime} ; \widetilde{f}^{*}\right)$ of 0 , whose size depends on the choice of $O\left(\left(z^{\prime}, 0\right)\right)$ and the choice of $\tilde{f}^{*}$. (d) $\hat{f}(\bar{\xi}, \bar{\tau})$ is any point in $\mathcal{F}(\bar{\xi}, \bar{\tau})$.

We next show that

$$
q_{l}\left(w^{\prime}, \lambda\right) \equiv q_{0}\left(w^{\prime}, \lambda\right) h_{l}^{*}\left(w^{\prime}, \lambda, q_{0}\left(w^{\prime}, \lambda\right)\right)
$$

for any $\left(w^{\prime}, \lambda\right) \approx 0$ and for each $l<n+1$. Indeed, this can be argued as follows:

Let $z_{0}^{\prime} \in \mathbf{C}^{n}$ be chosen as above such that $\lambda\left(z^{\prime}\right)=\overline{\widetilde{f} *}\left(\overline{z^{\prime}}, 0\right)$ is an open mapping from $O\left(\left(z_{0}^{\prime}, 0\right)\right) \cap Q_{0}$ to $\mathbf{C}^{n}$. Fix each $z^{\prime} \in O\left(\left(z_{0}^{\prime}, 0\right)\right) \cap Q_{0}$ and consider the map $H$ which sends each $\xi(\approx 0) \in \mathbf{C}^{n}$ to $\left(\hat{f}^{*}\left(\bar{\xi}, \overline{\mu\left(z^{\prime}, \xi\right)}\right), f_{n+1}\left(\bar{\xi}, \overline{\mu\left(z^{\prime}, \xi\right)}\right)\right)$. Since $\left(\hat{f}^{*}(\bar{\xi}, \bar{\tau}), f_{n+1}(\bar{\xi}, \bar{\tau})\right) \in Q_{\left(\tilde{f}^{*}\left(z^{\prime}, 0\right), 0\right)}^{\prime}$, by Lemma 8.1, and from the fact $\overline{\left(\xi, \mu\left(z^{\prime}, \xi\right)\right)} \in Q_{\left(z^{\prime}, 0\right)}$, we see that $H$, which sends each $\xi$ into $Q_{\left(\tilde{f}^{*}\left(z^{\prime}, 0\right), 0\right)}^{\prime}$, is a finite to one map. Next, let $\pi^{*}$ be the natural projection from $\mathbf{C}^{n+1}$ to its first $n$-copies of $\mathbf{C}$. Then, it follows easily that $\pi^{*} \circ H$ is an open mapping. This tells that for each $z^{\prime} \approx z_{0}^{\prime}$, (10.5) holds for an open subset of $w^{\prime} \in \mathbf{C}^{n}$. Hence, from the uniqueness theorem of holomorphic functions, we conclude that (10.5) holds identically.

Now, as in [7], by applying the basic theory of ODE to

$$
q_{l}\left(w^{\prime}, \lambda\right)=q_{0}\left(w^{\prime}, \lambda\right) h_{l}^{*}\left(w^{\prime}, \lambda, q_{0}\right)
$$

and by using the initial condition $q_{0}\left(w^{\prime}, 0\right)=G\left(w^{\prime}, 0,0\right)=0$, we conclude that $q_{0} \equiv 0$. This contradicts the finite D'Angelo type assumption for $M_{1}$.

We now prove the following preservation principle for our map $\mathcal{F}$ :

Lemma 10.2. After shrinking $\mathcal{P}, \mathcal{F}(\mathcal{P} \cap D)$ and $\mathcal{F}\left(\mathcal{P} \cap D^{c}\right)$ stay in different sides of $M_{2}$. 


\section{XIAOJUN HUANG}

Proof of Lemma 10.2: We will still use the reflection function $G(\mathcal{F}, \lambda)$ introduced in Lemma 7.1. Let

$$
\rho^{*}(z, \bar{w})=1 / N \operatorname{Re}\left(\sum_{\tilde{f}(w) \in \mathcal{F}(z)}\left(\overline{f_{n+1}(w)}-G\left(\mathcal{F}(z), \overline{\tilde{f}^{*}(w)}\right)\right)\right),
$$

where $N$ is the generic counting number of $\mathcal{F}(z)$. Then by Lemma 7.1 and the Riemann extension theorem, we see that $\rho^{*}(z, \bar{w})$ is holomorphic in $(z, \bar{w})$. In particular, $\rho^{*}(z, \bar{z})$ is real analytic near 0 . We claim that $\rho^{*}(z, \bar{z})$ is $<0$, when $\mathcal{F}(z) \subset \Omega^{+} ;>0$ when $\mathcal{F}(z) \subset \Omega^{-}$; and $=0$ when $\mathcal{F}(z) \subset M_{2}$ (see Lemma 8.3 (a) for related notations). In fact, for each fixed $\widetilde{f}(z) \in \mathcal{F}(z)$, we have

$$
\begin{gathered}
\operatorname{Re}\left(\overline{f_{n+1}(z)}-G\left(\widetilde{f}, \overline{\widetilde{f}^{*}(z)}\right)\right)=\operatorname{Re} \widetilde{\rho_{2}}\left(\widetilde{f}, \overline{\widetilde{f}(z)^{*}}\right) \\
\left.\left.=\rho_{2}(\widetilde{f}, \overline{\widetilde{f}(z)}) \operatorname{Reh}_{2}(\widetilde{f}(z), \overline{\widetilde{f}(z)})\right)=\rho_{2}(\widetilde{f}, \overline{\widetilde{f}(z)})\right)(1+o(\|\widetilde{f(z)}\|),
\end{gathered}
$$

where $\widetilde{\rho}_{2}=\rho_{2} h_{2}$. Thus, the claim follows from Lemma $8.3(\mathrm{v})$.

Next, applying Lemma 10.1, one sees that

$$
\rho^{*}=2 \operatorname{Re}\left(f_{n+1}(z)\right)+o(|z|)=\operatorname{Re}\left(g(0) z_{n+1}\right)+o(|z|)
$$

with $g(0) \neq 0$. Thus, $\mathrm{d}_{0} \rho^{*} \neq 0$. Therefore, $\rho^{*}$ serves as a real analytic defining function of $M_{1}$ near 0 . Without loss of generality, let us assume that $\rho^{*}(z, \bar{z})<0$ for $z \in D$. Then $\rho^{*}(z, \bar{z})>0$ for $z \in D^{c}$. Now, by making $\mathcal{P}$ small, the above argument shows that $\mathcal{F}(D \cap \mathcal{P}) \subset \Omega^{+}$and $\mathcal{F}\left(D^{c} \cap \mathcal{P}\right) \subset \Omega^{-}$.

Remark 10.3: In what follows, we will write $\Omega=\Omega^{+}$. Then we observe that the above results give the fact that $\mathcal{F}(D) \subset \Omega$ and $\mathcal{F}\left(D^{c}\right) \subset \Omega^{c}$ with $\Omega^{c}=U^{\prime}(0) \backslash\left(\Omega \cup M_{2}\right)$.

We now are ready to show that $f$ admits a proper holomorphic extension to the $D$-side:

Proof of Theorem $D(b)$ : With the above notation, let $B$ be a sufficiently small ball centered at 0 . Let $U^{*}$ to be the connected component of the set 


\section{SCHWARZ REFLECTION PRINCIPLE}

$\{z \in B \cap D: f(z) \notin f(\partial B \cap D)\}$ such that $f\left(U^{*}\right)$ contains $\Omega \cap O(0)$ with $O(0)$ sufficiently small. Then, Lemma 10.2 and Lemma 8.3 (i.v) indicate that $U^{*}$ is a bounded domain which contains a small piece of $M_{1}$ near the origin as part of its smooth boundary. By a well-known result (see [14], for example), one can see that $f$ is proper from $U^{*}$ to its image. Hence, after taking $D=U^{*}$ and $\Omega=f\left(U^{*}\right)$, we see that $f$ is proper from $D$ to $\Omega$.

As another application of the Hopf lemma, we have the following proposition, which will be used in $\S 11$.

Proposition 10.4. Assume all the previous notation. Let $a, b \in \mathbf{C}^{n+1}$ be sufficiently close to the origin. If $Q_{\mathcal{F}(a)}^{\prime}=Q_{\mathcal{F}(b)}^{\prime}$, then $Q_{a}=Q_{b}$.

Proof of Proposition 10.4: Let $a$ and $b$ be as in the hypothesis of the proposition. We then need to show that $Q_{a} \cap \widetilde{\mathcal{P}}=Q_{b} \cap \widetilde{\mathcal{P}}$. To this aim, we consider the function $\widetilde{\rho_{2}}\left(\mathcal{F}(z), \overline{\tilde{f}^{*}(\omega)}\right)$, for any given $\widetilde{f}(\omega) \in \mathcal{F}(\omega)$. By Lemma 7.1, $\widetilde{\rho_{2}}\left(\mathcal{F}(z), \overline{\widetilde{f}^{*}(\omega)}\right)$ is well defined and holomorphic in $(z, \lambda)$ with $\lambda=\overline{\widetilde{f}^{*}(\omega)}$ for $(z, \lambda) \in O_{z}(0) \times O_{\lambda}(0)$. Hence, $\widetilde{\rho_{2}}\left(\mathcal{F}(z), \widetilde{\tilde{f}^{*}(\omega)}\right)=c z_{n+1}+c \bar{\omega}_{n+1}+$ $O(|z||\lambda|)+o(|\lambda|)$ for some $c \neq 0$, by Lemma 10.1. Thus, for each $\omega$ sufficiently close to 0 , since $|\widetilde{f}(\omega)| \approx 0$, the implicit function theorem indicates that $\widetilde{\rho_{2}}\left(\mathcal{F}(z), \widetilde{f^{*}(\omega)}\right)=0$ defines a connected complex hypersurface $W_{\omega}$ in some small neighborhood $U^{\#}$ of 0 , whose size is independent of the parameter $\omega$ once $|\omega|$ is sufficiently small. Notice, by Lemma 8.1, that $\mathcal{F}\left(Q_{\omega}\right) \subset Q_{\tilde{f}(\omega)}^{\prime}$ near 0 . We see that $Q_{\omega} \cap U^{\#} \subset W_{\omega}$. Thus it follows that $W_{\omega}=Q_{\omega}$ in $U^{\#}$ when $|\omega|<<1$; for $Q_{\omega}$ is also a connected complex submanifold of codimension 1 in $\widetilde{\mathcal{P}}$.

Now, if $Q_{\mathcal{F}(b)}^{\prime}=Q_{\mathcal{F}(a)}^{\prime}$, we then conclude that $\widetilde{\rho}_{2}\left(\mathcal{F}(z), \overline{\mathcal{F}^{*}(b)}\right)=0$ and $\widetilde{\rho}_{2}\left(\mathcal{F}(z), \overline{\mathcal{F}^{*}(a)}\right)=0$ define the same variety. Hence, when $a, b \approx 0$, we conclude that $Q_{a}=Q_{b}$; for both of them have a small open subset of $W_{a} \cap$ $U^{\#}=W_{b} \cap U^{\#}$ in common. This completes the proof.

\section{$\S 11$. Completion of the Proof of Theorem C}




\section{XIAOJUN HUANG}

We now proceed to the proof of Theorem D (c), i.e, Theorem C. In this section, we let $n=1$. For simplicity, we retain all the notation which we have set up in the previous sections of Part II.

Lemma 11.1. (a) Let $M_{1}$ and $D$ be as before. There is an analytic variety $V \supset \mathcal{B}$ such that (i) $V \cap Q_{0}=\{0\}$, and (ii) for each sufficiently small $t>0$, there is a disk, denoted by $\Delta(t)\left(\subset \mathbf{C}^{1}\right)$, so that $V \cap\left\{\left(z_{1},-t\right): z_{1} \in \mathbf{C}^{1}\right\} \subset$ $\Delta(t) \times\{-t\} \subset D$. Similar statement also holds for the pair $M_{2}$ and $\Omega$.

(b) Let $V$ be chosen as in (a). Then $\mathcal{E} \subset V$.

Proof of Lemma 11.1: Let $M_{1}$ be defined by the following normalized equation:

$$
z_{2}+\overline{z_{2}}+\sum_{j=0}^{\infty} \widetilde{\phi_{j}}\left(z_{1}, \overline{z_{1}}\right) z_{2}^{j}=0 ; \text { or } z_{2}+\overline{z_{2}}+\sum_{j=0}^{\infty} \overline{\widetilde{\phi_{j}}}\left(\overline{z_{1}}, z_{1}\right) \overline{z_{2}^{j}}=0
$$

For each $b=\left(b_{1}, b_{2}\right) \approx 0, A_{b}=\left\{\left(w_{1}, w_{2}\right): Q_{w} \cap \widetilde{\mathcal{P}}=Q_{b} \cap \widetilde{\mathcal{P}}\right\}$. As did in Lemma 8.3, one sees that $w_{2}=b_{2}$. Now, it is easy to see that $Q_{\underline{w}}$ can be defined by $z_{2}=-\overline{b_{2}}-\sum_{j=0}^{\infty} \overline{\widetilde{\phi}_{j}}\left(\bar{w}_{1}, z_{1}\right){\overline{b_{2}}}^{j}$, where the notation $\overline{\widetilde{\phi}}$ is the same as explained before. Write $\overline{\widetilde{\phi}_{j}}\left(\overline{w_{1}}, z_{1}\right)=\sum_{\alpha>0} \overline{\Xi_{j \alpha}}\left(\overline{w_{1}}\right) z_{1}^{\alpha}$. Then $z_{2}=-\overline{b_{2}}-\sum_{\alpha} \sum_{j} \bar{\Xi}_{j \alpha}\left(\bar{w}_{1}\right){\overline{b_{2}}}^{j} z_{1}^{\alpha}$. So, the equation: $Q_{w}=Q_{b}$ can be written as $\sum_{j} \bar{\Xi}_{j \alpha}\left(\bar{w}_{1}\right){\overline{b_{2}}}^{j}=\sum_{j} \bar{\Xi}_{j \alpha}\left(\bar{b}_{1}\right){\overline{b_{2}}}^{j}$ or $\sum_{j} \Xi_{j \alpha}\left(w_{1}\right) b_{2}^{j}=\sum_{j} \Xi_{j \alpha}\left(b_{1}\right) b_{2}{ }^{j}$, for any $\alpha$.

Choose $k_{0}$ to be the smallest integer so that for some $\alpha_{0}, \Xi_{0 \alpha_{0}}\left(w_{1}\right)=$ $a^{*} w_{1}^{k_{0}}+o\left(\left|w_{1}\right|^{k_{0}}\right)$ with $a^{*} \neq 0$.

Write $P\left(w_{1}, b_{2}\right)=\Xi_{0 \alpha_{0}}\left(w_{1}\right)+\sum_{j>0} \Xi_{j \alpha_{0}}\left(w_{1}\right) b_{2}^{j}$. For $\left(b_{1}, b_{2}\right)$ close to the origin, we define

$$
V=\left\{\left(b_{1}, b_{2}\right): \text { for some } w_{1}^{0}, \quad P\left(w_{1}^{0}, b_{2}\right)=P\left(b_{1}, b_{2}\right), \frac{\partial P}{\partial w_{1}}\left(w_{1}^{0}, b_{2}\right)=0\right\} .
$$

Then as in the proof of Lemma 9.1, one can easily verify that $Q_{0} \cap V=\{0\}$ and $\mathcal{B} \subset V$.

We next claim that $V$ possesses the other properties claimed in the lemma.

To see that, we let

$$
V^{*}=\left\{\left(b_{1}, b_{2}, w_{1}\right) \in O(0): P\left(w_{1}, b_{2}\right)=P\left(b_{1}, b_{2}\right), \frac{\partial P}{\partial w_{1}}\left(w_{1}, b_{2}\right)=0\right\} .
$$




\section{SCHWARZ REFLECTION PRINCIPLE}

Then $V$ is the projection of $V^{*}$ to the first two copies of $\mathbf{C}^{1}$. This projection map is obviously finite to one near the origin and thus is locally proper. Meanwhile, for each $b_{2}$, from $\frac{\partial P\left(w_{1}, b_{2}\right)}{\partial w_{1}}=0$, we can solve out only finitely many $w_{1}$ 's with $\left|w_{1}\right|$ small. Moreover, for any solution $w_{1}$, we can easily see that $\left|w_{1}\right| \lesssim\left|b_{2}\right|^{\frac{1}{k_{0}-1}}$. Thus it follows that there are also only finitely many $b_{1}$ 's so that $P\left(w_{1}, b_{2}\right)=P\left(b_{1}, b_{2}\right)$ with $\frac{\partial P\left(w_{1}, b_{2}\right)}{\partial w_{1}}=0$. Furthermore, one can also see that $\left|b_{1}\right|<\left|b_{2}\right|^{1 / k_{0}}$. Meanwhile, it is also easy to see that $V \cap\left(\mathbf{C}^{1} \times\left\{b_{2}\right\}\right) \subset \Delta_{\left|b_{2}\right|^{*}} \times\left\{b_{2}\right\}$ for $\left|b_{2}\right|$ sufficiently small. Here $\Delta_{r}$ is used to stand for the disk in $\mathbf{C}^{1}$ with center at the origin and with radius $r, \alpha^{*}$ is a constant between $\frac{1}{k_{0}+1}$ and $\frac{1}{k_{0}}$. By our choice of $k_{0}$, it follows easily for sufficiently small $\left|b_{2}\right|$, that $\Delta_{\left|b_{2}\right|^{\alpha^{*}}} \times\left\{b_{2}\right\} \subset D$ when $b_{2}<0$. This completes the proof of of Lemma 11.1 (a).

(b). We first observe that in the two dimensional case, $\mathcal{E}$ is exactly the branch locus of the irreducible Weierstrass polynomial defining $f_{1}$. Hence for $z \in \mathcal{E}$, there is always a sequence $\left\{z_{j}\right\} \rightarrow z$ such that $\# \pi^{-1}(z)<\# \pi^{-1}\left(z_{j}\right)$. So, the exact argument as in Lemma 9.2 (a) shows that $\mathcal{F}(\mathcal{E}) \subset \mathcal{B}^{\prime}$. We next show that $\mathcal{E} \cap O(0) \subset V$, where $V$ is as defined in (a). To this aim, we assume, without loss of generality, that $\mathcal{E} \neq \emptyset$. Let $\cup_{j} \mathcal{E}_{j}$ be the union of the irreducible components of $\mathcal{E}$ near 0 . We notice that each $\mathcal{E}_{j}$ then must be of dimension 1 at 0 . Now, since $\mathcal{F}\left(\mathcal{E}_{j}\right) \subset \mathcal{B}^{\prime}$ is also an analytic variety of codimension 1 at 0 (Lemma 8.3 (iv)), the assertion in (a) indicates that $\mathcal{F}\left(\mathcal{E}_{j}\right) \cap \Omega \neq \emptyset$ near 0 . Therefore, it follows from Lemma 10.2, that $\mathcal{E}_{j} \cap D$ is not empty in any neighborhood of 0 . Now, pick any point $z \in \mathcal{E}_{j} \cap D$, which is sufficiently close to 0 . Then, as above, we can find a sequence $z_{j} \rightarrow z$ and $\left\{\eta_{j}\right\},\left\{\xi_{j}\right\}$ with $\xi_{j}, \eta_{j} \in \mathcal{F}\left(z_{j}\right) \eta_{j} \neq \xi_{j}$, but $\xi_{j}, \eta_{j} \rightarrow w_{0} \in \Omega$. By making $z$ sufficiently close to 0 , we can assume by Theorem D (b), that $f\left(z_{0}\right)=w_{0}$ for some $z_{0}$ close to 0 . Moreover, $f$ is proper from a small neighborhood $U$ of $z_{0}$ and $\bar{U} \cap f^{-1}\left(w_{0}\right)=\left\{z_{0}\right\}$. Since $f$ is single valued, there are two distinct points $a_{j}, b_{j} \in U$ such that $f\left(a_{j}\right)=\xi_{j}$ and $f\left(b_{j}\right)=\eta_{j}$. Now, Proposition 10.4 indicates that $a_{j} \in A_{b_{j}}$. Since $a_{j}, b_{j}$ clearly converge to $z_{0}$ as $j \rightarrow \infty$, we conclude that $z_{0} \in \mathcal{B}$. Again by Lemma 8.1 and Proposition 10.4, we see that $z_{0}, z \in \mathcal{B}$. By the arbitrariness of the choice of $z$ and by what we 


\section{XIAOJUN HUANG}

obtained in (a), we conclude that $\mathcal{E}_{j}$ has an open subset contained in $\mathcal{B}$ and thus in $V$. From the uniqueness of analytic varieties, it follows that $\mathcal{E}_{j} \subset V$ for any $j$.

We now are finally ready to complete the proof of Theorem C.

Proof of Theorem $C$ : The idea is to use the Hartogs extension theorem. By Lemma 11.1, we know that $\mathcal{E}$ is defined by an equation of the form: $z_{1}^{N}+\sum_{j=0}^{N-1} a_{j}\left(z_{2}\right) z^{j}=0$. Let $t$ be a small positive number and fix a small $\delta$

Now, using the hypothesis on $\mathcal{E}$ and shrinking $\delta$ if necessary, we can find small $R>0$ such that $\mathcal{E} \cap\left(\mathbf{C}^{1} \times \Delta_{\delta}\right) \subset \subset\left(\Delta_{\frac{1}{2} R} \times \Delta_{\delta}\right)$ Write

$$
\Omega_{0}=\left(\Delta_{R} \times\left(B_{\eta}(-t)\right)\right) \cup\left(\left(\Delta_{R} \backslash \overline{\Delta_{\frac{1}{2} R}}\right) \times \widetilde{B}(t)\right) .
$$

Here, $(0 \notin) B_{\eta}(-t)$ is a small disk centered at $-t$ with radius $\eta$ and $\widetilde{B}(t) \subset \Delta_{\delta}$ is another disk which contains $B_{\eta}(-t)$ and 0 .

Then, it is easy to see that any (closed) loop in $\Omega_{0} \backslash \mathcal{E}$ based at certain $z_{0} \in\left(\Delta_{R} \times\left(B_{\eta}(-t)\right)\right) \backslash \mathcal{E}$ can be deformed, relative to the base point and without cutting $\mathcal{E}$, to a loop in $\left(\Delta_{R} \times B_{\eta}(-t)\right) \backslash \mathcal{E}$, by our above choices.

Claim 11.2. Suppose that for a sufficiently small $\eta$, $f$ can be extended holomorphically to $\Delta_{R} \times B_{\eta}(-t)$. Then $f$ admits a holomorphic extension across 0 .

Proof of Claim 11.2: Let $\Omega_{0}$ be as defined above. We then notice that the holomorphic hull of $\Omega_{0}$ is $\Delta_{R} \times \widetilde{B(t)}$. In fact, for any $\phi \in \operatorname{Hol}\left(\Omega_{0}\right)$, the following Cauchy integral gives the holomorphic extension of $\phi$ to $\Delta_{R} \times \widetilde{B}(t)$ :

$$
\frac{1}{2 \pi i} \int_{|\xi|=\widetilde{R}} \frac{\phi\left(\xi, z_{2}\right)}{\xi-z_{1}} \mathrm{~d} \xi \text {, with } \widetilde{R} \rightarrow R \text {. }
$$

Now, since any loop in $\Omega_{0} \backslash \mathcal{E}$ can be deformed to a loop in $\Delta_{R} \times\left(B_{\eta}(-t)\right)$ without cutting $\mathcal{E}$, by the monodromy theorem, one sees that under the hypothesis, $f$ can be extended holomorphically to $\Omega_{0}$. Hence, $f$ admits a holomorphic extension to a small neighborhood of the origin. This completes the proof of Claim 11.2. 


\section{SCHWARZ REFLECTION PRINCIPLE}

We next need the following simple topological fact, whose proof is easy and is left to the reader:

Fact: Assume that $t, \eta$ are sufficiently small. Write $z^{0}=(\xi,-t) \in$ $\Delta_{R} \times\{-t\} \backslash \mathcal{E}$ for a certain $\xi$, where $R$ is as chosen before. Then any loop in $\Delta_{R} \times B_{\eta}(-t) \backslash \mathcal{E}$, which is based on $z^{0}$, can be deformed, relative to the base point and without cutting $\mathcal{E}$, into $\Delta_{R} \times\{-t\}$. Here, without loss of generality, we assume that the variety $\mathcal{E}$, which has dimension at most 1 , is smooth away from 0 .

Let $t$ be chosen as in the above fact. By Lemma 11.1, the finite set $\mathcal{E} \cap\left\{\Delta_{R} \times\{-t\}\right\}$ stays in $\Delta(t) \cap\left\{\mathbf{C}^{1} \times\{-t\}\right\} \subset \subset D$, where $\Delta(t)$ is as chosen in Lemma 11.1 (a).

On the other hand, since $f$ is holomorphic in a small neighborhood of $\Delta(t) \times\{-t\}$, by the monodromy theorem, we see that $f$ admits a holomorphic extension to a neighborhood of $\Delta_{R} \times\{-t\}$. Hence by the above fact,

the monodromy theorem, and Claim 11.2, we conclude that $f$ admits an extension across 0 .

The proof of Theorem $\mathrm{C}$ is complete now.

\section{REFERENCES}

[1] M.S. Baouendi, S. Bell, and L. P. Rothschild, Mappings of three dimensional CR manifolds and their holomorphic extension, Duke Math. J. 56 (1988), 503-530.

[2] M. S. Baouendi, C. H. Chang, and F. Treves, Microlocal hypoanalyticity and extension of CR functions, J. Diff. Geom. 18 (1983), 331-391.

[3] M. S. Baouendi, H. Jacobowitz, and Treves, On the real analyticity of CR mappings,, Ann. of Math. 122 (1985), 365-400.

[4] M. S. Baouendi, X. Huang, and L. Rothschild, Regularity of CR mappings between algebraic hypersurfaces, Invent. Math, (to appear). 


\section{XIAOJUN HUANG}

[5] M. S. Baouendi, X. Huang, and L. Rothschild, Nonvanishing of the differential of holomorphic mappings at boundary points, Math. Research Letters 2 (1995), 737-750.

[6] M.S. Baouendi and L.P. Rothschild, Germs of CR maps between real analytic hypersurfaces, Invent. Math. 93 (1988), 481-500.

[7] M.S. Baouendi and L. P. Rothschild, Geometric properties of smooth and holomorphic mappings between surfaces in complex spaces, J. Diff. Geom. 31 (1990), 473-499.

[8] M. S. Baouendi and L. P. Rothschild, Remarks on the generic rank of a CR mappings, J. Geom. Anal. 2 (1990), 1-9.

[9] M. S. Baouendi and F. Treves, About the holomorphic extensions of CR functions on real hypersurfaces, Duke Math. J. 51 (1984), 77-107.

[10] S. Bell, Analytic hypoellipticity of the $\bar{\partial}$-Neumann problem and extendibility of holomorphic mappings, Acta Math. 147 (1981), 109116.

[11] S. Bell, Biholomorphic mappings and the $\bar{\partial}$-problem, Ann. of Math. 114 (1982), 103-113.

[12] S. Bell, Local regularity of CR homeomorphisms, Duke Math. J. 57 (1988), 295-300.

[13] S. Bell, CR mappings between hypersurfaces in $\mathbf{C}^{n}$, (preprint).

[14] S. Bell and D. Catlin, Regularity of CR mappings, Math. Z. (1988), 357-368.

[15] S. Bell and R. Narasimhan, Proper holomorphic mappings of complex spaces, EMS 69, Several Complex Variables (edited by W. Barth and R. Narashimhan ), Springer-Verlag, 1990.

[16] E. M. Chirka, Complex analytic sets, Encyclopaedia of Mathematical Sciences (in Several Complex Variables), Springer-Verlag, 1985.

[17] E. Bierstone and P. D. Milman, Semianalytic and subanalytic sets, Inst. Hautes Etudes Sci. Publ. Math 93 (1988), 481-500.

[18] J. P. D'Angelo, Real hypersurfaces, order of contact, and applications, Ann. of Math. 115 (1982), 615-637. 


\section{SCHWARZ REFLECTION PRINCIPLE}

[19] K. Diederich and J. Fornaess, Proper holomorphic mappings between real analytic pseudoconvex domains in $\mathbf{C}^{n}$, Math. Ann. 282 (1988), 681-700.

[20] K. Diederich and J. Fornaess, Applications holomorphes propres entre domaines a bord analytic reel, C.R. Acad. Sci. Paris 307 (1988), 321- 324 .

[21] K. Diederich and J. Fornaess, Biholomorphic maps between certain real analytic domains in $\mathbf{C}^{2}$, Math. Ann. 245 (1979), 255-272.

[22] K. Diederich, J. Fornaess, and Z. Ye, Biholomorphisms in Dimension 2, J. Geom. Anal. 4 (1994), 539-552.

[23] K. Diederich and S. Pinchuk, Lecture notes and lecture on the boundary behavior of biholomorphic maps between real analytic domains in $\mathbf{C}^{2}$ by $\mathrm{S}$. Pinchuk at the University of Chicago (March, 1995); or by K. Diederich at Zurich (August, 1994).

[24] K. Diederich and S. Webster, A reflection principle for degenerate real hypersurfaces, Duke Math. J. 47 (1980), 835-843.

[25] C. Fefferman, The Bergman kernel and biholomorphic mappings of pseudoconvex domains, Invent. Math. 26 (1974), 1-65.

[26] F. Forstneric, A survey on proper holomorphic mappings, Proceeding of Year in SCVs at Mittag-Leffler Institute, Math. Notes 38, Princeton University Press, Princeton, N.J., 1992.

[27] R. C. Gunning and H. Rossi, Analytic Functions of Several Complex Variables, Prentice-Hall, Englewood Cliffs, N. J., 1965.

[28] X. Huang, Geometric Analysis in Several Complex Variables, Ph.D Thesis, Washington University, August, 1994.

[29] X. Huang, Regularity of holomorphic correspondences and applications to the mappings problems, preprint, August, 1995 (MSRI Preprint Series No. 094-95).

[30] X. Huang and Y. Pan, Proper holomorphic mappings between real analytic domains in $\mathbf{C}^{n}$, Duke Math. J., (to appear).

[31] H. Lewy, On the boundary behavior of holomorphic mappings, Atti Acad Naz Lincei Rend. Cl. Fis. Mat. Natur (1977), 1-8. 


\section{XIAOJUN HUANG}

[32] Y. Pan, On a characterization of the finite multiplicity of a $C R$ mapping, (preprint).

[33] S. I. Pinchuk, A boundary uniqueness theorem for holomorphic functions of several complex variables, Math. Zametki 15 (1974), 205212 .

[34] S. I. Pinchuk, On proper holomorphic mappings of strictly pseudoconvex domains, Siberian Math. J. 15 (1974), 909-917.

[35] S. I. Pinchuk and Tsyganov, Smoothness of CR mappings between strongly pseudoconvex hypersurfaces, Akad. Nauk. SSSR 53 (1989), 1120-1129.

[36] J. M. Trepreau, Sur le prolongement des fonctions CR definies sur une hypersurface reel de classe $C^{2}$ dans $\mathbf{C}^{n}$, Invent. Math. 83 (1986), 583-592.

[37] V.S. Vladimirov, Methods of the Theory of Functions of Many Variables, M. I. T. Press, Cambridge, Mass, 1966.

[38] S. Webster, On the mapping problem for algebraic hypersurfaces, Invent. Math. 43 (1977), 53-68.

[39] S. Webster, Biholomorphic mappings and the Bergmann kernels off diagonal, Invent. Math. 51 (1979), 155-169.

[40] H. Whitney, Complex Analytic Varieties, Addison-Wesley Publishing Company, 1972.

Department of Mathematics, The University of Chicago, Chi -CAgo, IL 60637, And MSRI, 1000 Centennial Drive, Berkeley, CA 94726.

E-mail address: xhuang@math.uchicago.edu; xhuang@msri.org 